Ecker, Y. \& Bar-Anan, Y. (in press). Conceptual Overlap between Stimuli Increases Misattribution of Internal Experience. Journal of Experimental Social Psychology

\title{
Conceptual Overlap between Stimuli Increases Misattribution of Internal Experience
}

\author{
Yael Ecker \\ Department of Psychology, Ben-Gurion University in the Negev, Be'er Sheva, \\ Israel \\ Coller School of Management, Tel-Aviv University, Israel \\ and \\ Yoav Bar-Anan \\ Department of Psychology, Ben-Gurion University in the Negev, Be'er Sheva, \\ Israel \\ School of Psychological Science, Tel-Aviv University, Israel
}

Contact details:

Yael Ecker -- Coller School of Management, Tel-Aviv University, Israel 69978; e-mail:

yae.ecker@gmail.com.

Yoav Bar-Anan - School of Psychological Sciences, Tel-Aviv University, Israel 69978; e-mail: baranan@tauex.tau.ac.il

Correspondence should be addressed to: Yael Ecker; Coller School of Management, Tel-Aviv University, Israel 69978; e-mail: yae.ecker@gmail.com.

Funding: this work was supported by the Israeli Science Foundation [grant number 779/16]; the United States-Israel Binational Science Foundation [grant number 2013214]; and Project Implicit, Inc., awarded to Y. B.-A. 


\title{
Conceptual Overlap between Stimuli Increases Misattribution of Internal Experiences Yael Ecker and Yoav Bar-Anan \\ Department of Psychology, Ben-Gurion University in the Negev, Be’er Sheva, Israel
}

\begin{abstract}
People make sense of their internal experiences by attributing them to objects (I am excited because he is here). This attribution decision influences one's opinion of the object (I like him). Misattribution occurs when the internal experience is triggered by one object (a prime), but attributed to another (a target). The present research used the Affect Misattribution Procedure to examine the influence of conceptual overlap between the prime and the target on misattribution of valenced internal experiences. For instance, would people misattribute the pleasure activated by a flower prime to a target flower more than to a person? We argue that the valence of the internal experience does not enter the attribution process alone, but is rather accompanied by semantic properties related to the object that generated the experience. Consequently, misattribution occurs more when prime and target are conceptually similar. Four experiments (Total $\mathrm{N}=3,183$ ) supported that prediction, using diverse primes and targets. The results suggest that the semantic properties of the internal experience help guide the attribution process.
\end{abstract}

Keywords: Misattribution; Attribution, Priming, Affect Misattribution Procedure, Implicit measures. 


\section{Conceptual Overlap between Stimuli Increases Misattribution of Internal Experience}

Attribution (Bem, 1972; Laird, 1974; Schwarz \& Clore, 1983) is the cognitive process

whereby people associate their internal experience (i.e., their thoughts and feelings) with an object that (they believe to have) elicited it (e.g., I am afraid because of the bulldog). The process of attribution involves three main components: an internal experience, the object that generated the internal experience, and the object to which the experience is eventually attributed. When the last two components refer to different objects, attribution is erroneous. Research has shown that people can misattribute internal experiences triggered by drugs, physical exercise, or scary situations (e.g., crossing a narrow bridge) to a person or a social situation (Schachter \& Singer, 1962; Reisenzein \& Gattinger, 1982; White, Fishbein, \& Rutsein, 1981). They can misattribute somatic symptoms to WIFI radiation (Bräscher, Raymaekers, Van den Bergh, \& Witthöft, 2017) or to premenstrual syndrome (Schnall, Abrahamson, \& Laird, 2002), and misattribute the pleasantness of a sunny day to a general satisfaction with life (Schwarz \& Clore, 1983).

According to "constructivist" models of emotion, attribution is a central process in the formation of emotional experiences (Russell, 2003; Schachter \& Singer, 1962). This approach commonly argues that people's internal experience has two fundamental dimensional properties, termed arousal and valence (Barrett, 2006; Russell, 2003). Emotional experience occurs when people's core affect at a given moment in time is latched on to an eliciting object or situation. For instance, when arousal is attributed to an attractive person, the resulting emotion might be infatuation. The same experience of arousal, when attributed to an annoying person, would produce the emotion irritability. Accordingly, research on misattribution has traditionally assumed that the objects of attribution are either valence (Murphy \& Zajonc, 1993) or arousal (Reisenzein \& Gattinger, 1982; Schachter \& Singer, 1962; White et al., 1981). 
More recently, research on misattribution has shown that people can misattribute semantic content (Blaison, Imhoff, Hühnel, Hess, \& Banse, 2012; Deutsch \& Gawronski, 2009; Hofmann \& Baumert, 2010; Imhoff, Schmidt, Bernhardt, Dierksmeier, \& Banse, 2011). For instance, in one study, participants judged whether Chinese ideographs seemed to evoke fear or anger, while exposed to angry and fearful faces as primes (Blaison et al., 2012). Participants in that research tended to judge targets that appeared after angry primes as angry more often than fearful, and targets that appeared after fearful primes as fearful more often than angry. Because fear and anger are both negative and arousing experiences, something other than arousal and valence caused that effect. Valence and arousal were attributed alongside specific semantic information triggered by the primes.

The semantic information that internal experiences carry might have an important role in the attribution process. A fundamental difficulty in generating accurate attributions concerns the multi-dimensionality and complexity of the situations (Quine, 1960). Any given situation contains numerous objects to which an experience can be attributed (e.g., am I excited because of this ice-cream? Because I am with the person I love?). Despite decades of research into affect attribution, scientists are still far from having a principled account of the determinants of attribution of experience to an object. In the present research, we examined whether semantic information guides attribution, leading to an increase in the effect of misattribution on judgment when the prime and the target share meaning.

Observing a kitten, for instance, might trigger positive valence, and the concepts cute and furry. The attribution process might then search for the object that activated an experience that includes both positive valence and the specific semantic information. In other words, attribution might prioritize objects that share larger conceptual overlap with the internal experience. 
Consequently, a larger conceptual overlap between a prime and a target would increase the likelihood that an internal experience triggered by the prime would be misattributed to the target. We define conceptual overlap, broadly, as the degree to which the prime and the target share the same superordinate categories, whether that category is based on external properties (e.g., yellow objects), a similar function (e.g., work equipment), or any other common categorization through which people understand their environment.

The degree of conceptual overlap can be roughly estimated according to the most concrete superordinate category that the two stimuli (the prime and the target) share. Daisies and lilies, for instance, are both flowers (a rather concrete category), whereas daisies and cats can only share a category as abstract as "living things". Therefore, the conceptual overlap between daisies and lilies is certainly larger than between daisies and cats.

No previous research on misattribution has manipulated the conceptual overlap between the prime and the target. Thus, although there is evidence that semantic properties can be misattributed, it is currently unknown whether they can guide attribution towards target objects that share a high conceptual overlap with the object that triggered the internal experience. For instance, it is currently unknown whether a negative internal experience caused by an aggressive dog is more likely to be misattributed to a cat (a target that has a high conceptual overlap with a dog) than to a baby.

Interestingly, past research that investigated misattribution has often used primes and targets from very distant conceptual fields (Dutton \& Aron, 1974; White et al., 1981). One possible reason for this choice is the assumption that, for misattribution to occur, participants must not suspect the prime of influencing their judgment of the target. A prime that seems similar to a target of judgment may be perceived as likely to influence judgment, and would thus 
raise suspicion that would eliminate misattribution (Bless \& Schwarz, 2010; Wegener \& Petty, 1995). However, more recent research, that measured misattribution using the Affect Misattribution Procedure (AMP) — a sequential priming task with snap judgment of multiple targets, found that misattribution does not decrease when participants are warned that the primes might influence their judgment (Payne, Cheng, Govorun, \& Stewart, 2005). Therefore, at least in such judgment contexts, conceptual overlap might increase misattribution by signalling to the individual that the attribution in question is correct.

Evidence for the importance of conceptual overlap in attribution comes from recent research that investigated the applicability of the internal experience triggered by a prime to the judgment question in the AMP (Ecker \& Bar-Anan, 2018). In this research, the target was kept constant, and the manipulation varied the conceptual overlap between the prime and the judgment question. The results suggested that primes had a larger influence on judgment when they had a larger conceptual overlap with the judgment question. For instance, when participants judged human faces, a dirty room prime that appeared briefly before the target had a larger influence on judgment when participants judged cleanliness than when they judged cuteness. The negative valence of the room decreased both cuteness and cleanliness ratings. However, when the judgment was specifically related to cleanliness (rather than only to the room's valence), that influence increased.

One plausible account for the increase in misattribution when the primes are more applicable to the judgment question is that, when making judgment, people expect the target to elicit mental content related to the judgment that they are trying to make. Consequently, prime content that has high conceptual similarity to the judgment signals that the attribution in question is correct. Applied to the present research question, it seems reasonable to assume that a prime 
stimulus that is conceptually similar to the target would elicit content that is relevant to the judgment of the target. The judgment is framed by two components: the judgment question and the judgment's target. The question "How pleasant is this object?" is unclear until the judge knows what the object is. For instance, judging the pleasantness of a food item is rather different than judging the pleasantness of a dog. Following that rationale, a prime that is similar to the target would be more likely to activate mental content relevant for the judgment of that target. Therefore, just like larger conceptual overlap between the prime and the judgment question increases the effect of misattribution on judgment, so would larger conceptual overlap between the prime and the judgment's target.

Because differences between target types (e.g., between people that belong to different social groups) in susceptibility to judgment bias are of social importance, clarifying the distinct influence of the target (as opposed to the judgment question) on misattribution is of great value. Moreover, the identity of the target might be more consequential to attribution in real life than that of the question. In real life, the judgment question is more often insinuated than expressed explicitly. People tend to extract the question from context (e.g., when asked to evaluate a colleague at the workplace, people might extract the question- "how well does she do her job?"), or default to a rather abstract judgment question of "how much do I like this person?". The target of judgment, on the other hand, is usually clear and well-defined, and thus has a higher potential for influencing judgment.

Importantly, there are also reasons to predict that high conceptual overlap between the prime and the target would decrease, rather than increase, misattribution. Research shows that, under certain conditions, prime-target similarity can cause contrast effects-judgment bias in the opposite direction to the prime's valence or trait. Specifically, in the commonly used paradigm in 
impression formation research—wherein judgment is unhurried and primes appear separately from the judgment task - primes cause contrast effects when perceived as comparable to the targets (Aarts \& Dijksterhuis, 2002; LeBoeuf \& Estes, 2004; Moskowitz \& Skurnik, 1999). Thus, although prime-judgment conceptual overlap increases in the influence of misattribution on judgment, prime-target conceptual overlap might not have a similar influence.

We used the AMP (Payne et al., 2005) to examine whether people are more likely to misattribute a valenced internal experience triggered by a prime to a target of judgment when the prime and the target have high (rather than low) conceptual overlap. As a sequential priming paradigm wherein people make snap judgments about multiple targets, the AMP allows a relatively easy manipulation of the relation between the prime and the target of judgment. Early research on misattribution has mostly used procedures that involve complex social situations, rich with social detail (e.g., Dutton \& Aron, 1974; White et al., 1981). Although high on ecological validity, these procedures do not allow for well-controlled variations in specific elements of the attribution process. For instance, when the prime is a narrow bridge that participants happen to cross (Dutton \& Aron, 1974), physical exercise (Cantor, Zillmann, \& Bryant, 1975), a sunny day (Schwarz \& Clore, 1983), or a gut-wrenching movie clip (White et al. 1981), it is difficult to manipulate the similarity between that prime and the target of judgment while holding other aspects of the situation constant. This might explain why, to date, little is known about the manner in which the relations between elements in the attribution process influence attribution.

Although doubts about the mechanism behind the priming effect in the AMP have been raised (Bar-Anan \& Nosek, 2012), there is currently good evidence that the effect is caused by misattribution of internal experiences triggered by the prime to the target (e.g., Gawronski \& Ye, 
2014, 2015; Oikawa, Aarts, \& Oikawa, 2011). There is currently no good evidence in support of any other account. In the last decade, the AMP has improved the understanding of misattribution in the context of quick judgment. It has been shown, for instance, that focusing on gut feelings increases misattribution (De Houwer \& Smith, 2013), that longer exposure to the target of judgment decreases misattribution (Payne, Hall, Cameron, \& Bishara, 2010), and that a sense of uncertainty about the source of one's internal experience increases misattribution (Ruys, Aarts, Papies, Oikawa, \& Oikawa, 2012). The AMP provides a well-controlled tool for a continuous and relatively reliable measurement of misattribution, in the context of a quick judgment after a brief exposure to the target of judgment. Using the AMP, we were able to investigate a fundamental, perhaps long-overdue question, namely, how the conceptual overlap between a prime and the target of judgment influences misattribution of valenced experiences.

\section{Experiment 1}

We measured misattribution using a modified version of the AMP (Payne et al., 2005). We manipulated both the prime and the target categories, creating two high-overlap combinations between prime and target and two low-overlap combinations. That allowed us to examine the influence of conceptual overlap beyond the particular prime or target categories. The primes in the AMP were pleasant and unpleasant people or pleasant and unpleasant landscapes. Participants judged the pleasantness of either people or landscapes. For participants who judged people, conceptual overlap between the prime and the target was high when the primes were people and low when the primes were landscapes. For participants who judged landscapes, conceptual overlap was high when the primes were landscapes and low when the 
primes were people. We examined whether priming (the misattribution effect) was stronger when the conceptual overlap between the primes and the targets was high compared to low. ${ }^{1}$

\section{Method}

\section{Participants}

Participants in all studies were volunteers at the Project Implicit research website (Nosek, 2005). Most of the visitors in the Project Implicit website participate in studies about implicit bias toward social groups and social issues. Additionally, a few hundred visitors a day register to a different section of the website and participate (voluntarily) in a variety of studies related to implicit social cognition. Those participants are randomly assigned to one of the studies available in the website and cannot enter the same study more than once. The weaknesses of this population is a relatively high dropout rate (see Footnote 2). This population also has some notable strengths in comparison to the samples of most studies published in social psychology. Most importantly, the population is heterogeneous: the range of ages is wide, and students are usually less than half of the population. Many participants did not study psychology, and are less experienced in study participation than the typical participant in psychological studies, or in other web-based samples (e.g., MTurk). Many published studies in social psychology are limited to student populations or to highly experienced participants. Just as those studies require generalization with replications conducted with more heterogeneous samples, future research could test whether the present findings generalize to the lab, where settings are better controlled.

\footnotetext{
${ }^{1}$ We report all data exclusions, manipulations, and measures, and how we determined our sample sizes. The decision to stop collecting data did not depend on the obtained results.
} 
1,011 participants completed the experiment ${ }^{2}$, out of which we excluded 60 participants who rated all targets in a certain block with the same response. Our version of the AMP included a 7 point judgment scale ${ }^{3}$, which we hoped would seem less monotonous and more reasonable to participants than a dichotomous judgment scale. Because participants had 7 response options, using the same response throughout an entire task block indicates that judgment did not occur. This was the only exclusion rule used in all the experiments reported in this paper. 951 entered the analysis (57\% women, $\left.M_{a g e}=33.87, S D_{a g e}=14.32\right)$. Based on calculations made using G*Power (Faul, Erdfelder, Lang, \& Buchner, 2007), the final sample size achieved 97\% power to detect a conceptual overlap effect of $\eta_{\mathrm{p}}{ }^{2}=.02$. We reasoned that $\eta_{p}{ }^{2}=.02$ was the smallest effect-size we could investigate with reasonable power, given our resources.

Design. The design was 2 (target category: landscapes, faces; between participants) X 2 (prime category: landscapes, people; between participants) X 2 (prime valence: positive, negative; within participants).

Materials. The prime stimuli ${ }^{4}$ were images of positive and negative landscapes and people. There were nine images of each type (36 in total; taken from online public domains and from the International Affective Picture System; Bradley \& Lang, 2007). Positive people were

\footnotetext{
${ }^{2}$ Because participation in experiments at the Project Implicit is voluntary, dropout during the experiment is relatively high (about $35 \%$ dropout in a typical experiment). The number of participants who did not complete all trials of the AMP were 455 (31\%), 476 (35\%), 454 (41\%), 417 (32\%), in Experiments 1- 4, respectively. High dropout rates might jeopardize random assignment and thus the internal validity of the experiment (Zhou \& Fishbach, 2016). However, this risk is probably minimal in this work because the manipulations give little cause for condition-dependent attrition. We did not analyze partial results and in the main text we discuss only participants who completed all the measures.

${ }^{3}$ In a separate project, we compared the continuous scale version of the AMP to the standard binary version. We summarized the results of this test in the supplementary material. In short, the psychometric qualities of the continuous scale version of the AMP are at least equal to those of the standard version

${ }^{4}$ We tested the prime stimuli in a separate study. In that study $(\mathrm{N}=367)$, each participant rated one fifth of the stimuli used throughout this research. The stimuli and their ratings appear in Table S10 in the supplementary material. In each prime category, the most negatively rated stimulus that we used as a positive prime was evaluated more favorably than the most positively rated stimulus that we used as negative primes, ps $<.001$, ds $=1.38,0.95$, $1.08,1.09,1.12$, and 2.18 for the categories food, dogs, flowers, men, women, and scenery, respectively.
} 
happy and good looking, whereas negative people were unaesthetic and had a negative emotional expression—either angry or sad. Positive landscapes were postcard-like, and negative landscapes were of landfill sites, cemeteries, and a polluting factory. The targets were 54 relatively neutral male faces collected from various sources (Minear \& Park, 2004; Nosek \& Banaji, 2001; Gawronski, Cunningham, LeBel, \& Deutsch, 2010; Payne, 2001), and 54 images of Israeli landscapes (fields, cities, parks and roads; taken from online public domains). The judgment scale was 1 - Very unpleasant, 2 - Moderately unpleasant, 3 - Slightly unpleasant, 4-Neutral, 5Slightly pleasant, 6 - moderately pleasant, and 7 - Very pleasant.

Procedure. In all the experiments, participants completed the task, programmed in Minno.js (Zlotnick, Dzikiewicz, \& Bar-Anan, 2015), over the Internet. The program assigned each participant randomly to one of the prime and target conditions. We instructed participants to ignore the first item presented in each trial, and rate whether the man [landscape] in the image that followed the first item was pleasant or unpleasant. Following Payne et al. (2005), we explicitly warned participants that the primes might distract them. Each trial started with the prime stimulus that appeared for $100 \mathrm{~ms}$, followed by a blank screen for $100 \mathrm{~ms}$, and then the target stimulus appeared for $100 \mathrm{~ms}$. A mask appeared at the end of the trial, until participants evaluated the target by hitting keys 1-7. The intertrial interval was $250 \mathrm{~ms}$. To increase participants' understanding of the task, the target stimuli in all the experiments always appeared in an orange frame, and the instructions at the beginning of each block explicitly requested participants to rate the second item, the one that appeared in an orange frame. The first block presented three practice trials. Then, participants completed three blocks of 36 trials (each prime stimulus appeared twice in each block). 


\section{Results}

We computed the priming score of each participant by subtracting the average rating score after negative primes from the average rating after positive primes. As shown in Table 1, priming was stronger when the prime and target categories were the same, rather than different.

For statistical inference, we conducted a 2 (target category: landscapes, people) x 2 (prime category: landscapes, people) x 2 (prime valence: positive, negative) repeated measures ANOVA on the average ratings. A main effect of prime valence, $F(1,947)=196.19, p<.001, \eta_{\mathrm{p}}{ }^{2}=.17$, reflected the expected priming effect. A main effect of target category, $F(1,947)=155.07, p<$ $.001, \eta_{\mathrm{p}}{ }^{2}=.14$, reflected more positive evaluations of landscapes compared to people. There was a prime category by prime valence interaction, $F(1,947)=12.35, p<.001, \eta_{\mathrm{p}}{ }^{2}=.01$, indicating stronger effect of prime valence by landscape primes, $F(1,491)=127.15, p<.001, \eta_{\mathrm{p}}{ }^{2}=.20$, than by people primes, $F(1,458)=54.95, p<.001, \eta_{\mathrm{p}}{ }^{2}=.11$. A two-way target category by prime valence interaction, $F(1,947)=72.86, p<.001, \eta_{\mathrm{p}}{ }^{2}=.07$, reflected a stronger effect of prime valence when the target category was landscape, $F(1,465)=173.96, p<.001, \eta_{\mathrm{p}}^{2}=.27$, than when the targets were people, $F(1,484)=25.68, p<.001, \eta_{\mathrm{p}}^{2}=.05$.

Most relevant to the research question, a reliable three-way interaction of prime category, target category and prime valence, $F(1,947)=22.28, p<.001, \eta_{\mathrm{p}}{ }^{2}=.02,90 \% C I[.01, .04]$, reflected a stronger priming effect when the prime and target categories were of the same category than when they were different. When the target category was landscapes, a prime valence by prime category interaction, $F(1,464)=23.39, p<.001, \eta_{\mathrm{p}}{ }^{2}=.05,90 \% C I[.02, .08]$, reflected a larger prime valence effect when the prime category was landscapes, $F(1,245)=$ 151.16, $p<.001, \eta_{\mathrm{p}}{ }^{2}=.38$, than when the prime category was people, $F(1,219)=39.47, p<$ $.001, \eta_{\mathrm{p}}{ }^{2}=.153$. When the target category was people, the same prime valence by target category 
interaction was not significant, $F(1,483)=1.26, p=.263, \eta_{\mathrm{p}}{ }^{2}<.01$, but the effect of prime valence was numerically larger when the prime category was people, $F(1,238)=16.68, p<$ $.001, \eta_{\mathrm{p}}{ }^{2}=.066$, than when the prime category was landscapes, $F(1,245)=9.20, p=.003, \eta_{\mathrm{p}}{ }^{2}=$ .04

Table 1.

Experiment 1: Mean Priming Scores by Target Category and Prime Category (standard deviation in parentheses).

\begin{tabular}{|c|c|c|c|}
\hline & & Targ & egory \\
\hline & & Landscapes & People \\
\hline & & $0.63 * *(0.81)$ & $0.09 *(0.45)$ \\
\hline & & $\mathrm{n}=246$ & $\mathrm{n}=220$ \\
\hline Prime Category & & & \\
\hline & & $0.29 * *(0.45)$ & $0.14 * *(0.52)$ \\
\hline & People & & \\
\hline & & $\mathrm{n}=246$ & $\mathrm{n}=239$ \\
\hline
\end{tabular}

Notes. All priming scores were significantly larger than zero with $p<.01$ (indicated by *), or $p<.001$ (indicated with $* *$ ).

\section{Discussion}

In viewing the results of Experiment 1, it is important to keep in mind the critical test for the conceptual overlap hypothesis is in the three-way interaction between target type, prime type, and valence. When looking only at one level of target type or prime type, the results can be biased by differences in the general potency of the specific primes and the susceptibility to priming of specific targets. For instance, when looking only at people as targets, we might not find that people primes caused more misattribution than scenery prime if the scenery primes are generally more potent (have a stronger valence). Similarly, when looking only at people as 
primes, we might not find a stronger effect on people targets if scenery targets have a general tendency to allow for more misattribution. The purpose of manipulating both prime type and target type simultaneously is to observe the influence of the match between prime and target beyond the tendency of specific primes and targets to encourage misattribution.

Experiment 1 found that high conceptual overlap between prime and target increased misattribution. When the targets were landscapes, participants misattributed more content of landscape primes than people primes. When targets were people, however, participants misattributed similarly content of landscape and people primes. This was a result of a generally higher potency of the landscape primes. Crucially, beyond the influence of specific prime and target types, participants made more attribution errors when primes were images of the same category as the targets.

Because semantic content (and not only general valence and arousal) participates in the attribution process, participants were able to use that content when making attribution decisions. For instance, after a positive scenery, the content pastoral directed participants to attribute their positive internal experience to a scenery target but not to a person. Importantly, perhaps because some mental content is general enough to fit both people and scenery (e.g.: charming, pleasant, beautiful), we found priming also in the low conceptual overlap conditions.

However, there is an alternative to the interpretation of the results as reflecting the effect of conceptual prime-target overlap. Perhaps the manipulation did not only vary conceptual overlap but also perceptual overlap — an overlap in the perceptual features of the prime and the target. Importantly, an increase in perceptual overlap may have caused an increase in perceptual confusion. Because the stimuli flash quickly, visual memory might blend visual aspects of the prime stimulus (e.g., a smile or a beautiful tree) with the target stimulus. In a well-known 
example of this effect, participants viewed a series of letters presented in different colors, and reported the letters and their color. The participants made conjunction errors - they perceptually misattributed visual features of one item (e.g., color) to other items that appeared next to it (Treisman \& Schmidt, 1982). In Experiment 2, we added conditions that reduced the likelihood of perceptual confusion.

\section{Experiment 2}

In Experiment 2, in addition to manipulating prime and target categories, we manipulated also the target's medium. Participants judged landscape or man images that were either photographs or abstract sketches. It is less likely that people would blend visual aspects of a live image into a sketch than blend two live images. Thus, if we find an effect for prime-target overlap when the primes are photographs and the targets are sketches, it would reduce the likelihood that only perceptual confusion, and not misattribution of conceptual content, is behind the effect. Perceptual confusion can cause people to erroneously perceive a perceptual feature of the prime stimulus (e.g., a smile) as a feature of the target stimulus. That is different than misattributing the internal experience that the prime triggers. By presenting primes and targets in different mediums, we hope to decrease to likelihood of perceptual confusion. Importantly, however, the conceptual overlap manipulation might still influence perceptual overlap when the targets are sketches, and the medium of target presentation might influence conceptual overlap. Therefore, the results wound not inform us whether conceptual overlap is more or less influential in attribution than perceptual overlap. The results could only help ascertain whether or not conceptual overlap plays a part in attribution.

Another change in Experiment 2 is that we manipulated the prime category within instead of between participants. The purpose of this change was to generalize our findings beyond a 
specific study design. For instance, it is possible that high prime-target conceptual overlap would not increase misattribution when participants are exposed to both types of primes (i.e., a withinparticipants design), because exposure to both prime types in the same task block would prompt participants to construe them as more conceptually similar than otherwise. Alternatively, exposure to both prime types might increase the conceptual overlap effect by emphasizing the difference between the two primes types (decreasing the conceptual overlap between them).

\section{Method}

Participants. 889 participants completed the experiment and 85 were excluded using the same exclusion rule as in Experiment 1 (participants who rated all targets in a certain block with the same response). $804(90 \%)$ participants entered the analysis $\left(62 \%\right.$, women, $M_{\text {age }}=37.93$, $\left.S D_{\text {age }}=15.09\right)$. This sample size yielded $94 \%$ power to detect a four-way interaction of prime category, target category, prime valence and target medium (sketch or photograph), with an effect size of $\eta_{\mathrm{p}}^{2}=.02$.

Design. The design was 2 (target category: landscapes, men; between participants) x 2 (target medium: sketch, image; between participants) x 2 (prime category: landscapes, men; within participants) x 2 (prime valence: positive, negative; within participants).

Materials. The prime stimuli were 24 photographic images of positive and negative landscapes and men (six of each valence-category combination; taken from the DepositPhotos image pool, from online public domains, and from the IAPS). The targets were six sets of 24 images. Two sets were 24 photographic images of men's faces and 24 photographic images of Israeli landscapes. Four other sets were of sketch images: two sets of 24 sketches of men's faces, and two sets of 24 landscape sketches (taken from the online image pool at depositphotos.com). We used two sets of sketches for each target category to increase the generalizability of the 
results. Thus, each participant was randomly assigned to rate one of four types of target sets: face sketches, landscape sketches, face photos, and landscape photos. Further, participants that rated sketch targets (either landscapes or faces), were randomly assigned to one of two sketch sets.

Procedure. Participants completed four blocks of 24 trials. Each block showed all the 24 targets in the set that was randomly assigned to the participant. We asked participants to judge how pleasant or unpleasant each landscape or face seemed to them.

\section{Results}

Table 2 shows the priming score in each condition. The table shows that priming was stronger when the prime and target categories were similar, for both sketch and photographic targets. Although the influence of conceptual overlap on priming seemed somewhat less pronounced when targets were sketches, it was still large.

For statistical inference, we conducted a 2 (target category: landscapes, men) x 2 (target medium: photograph, sketch) x 2 (prime category: landscapes, men) x 2 (prime valence: positive, negative) repeated measures ANOVA on the average ratings. A main effect of prime valence, $F(1,800)=536.64, p<.001, \eta_{\mathrm{p}}{ }^{2}=.40$, reflected the expected priming effect. A main effect of target category, $F(1,800)=208.36, p<.001, \eta_{\mathrm{p}}{ }^{2}=.21$, reflected more positive evaluations of landscapes compared to men. Similarly, a main effect of prime category, $F(1,800)=126.93, p<$ $.001, \eta_{\mathrm{p}}{ }^{2}=.14$, reflected more positive evaluations of the targets when primes were landscapes compared to men. A main effect of target medium, $F(1,800)=16.58, p<.001, \eta_{\mathrm{p}}{ }^{2}=.02$, reflected more positive evaluations of sketches compared to photographs. A number of interaction effects did not seem theoretically relevant to the present research question. We report these effects in the supplementary material. 


\section{Table 2.}

Experiment 2: Mean Priming Scores by Target Category, Prime Category, and Target Medium (standard deviation in parentheses).

\begin{tabular}{|c|c|c|c|c|c|}
\hline & & \multicolumn{2}{|c|}{ Sketch Targets } & \multicolumn{2}{|c|}{ Photographic Targets } \\
\hline & & \multicolumn{4}{|c|}{ Target Category } \\
\hline & & $\begin{array}{c}\text { Landscapes } \\
n=209\end{array}$ & $\begin{array}{c}\text { Men } \\
\mathbf{N}=\mathbf{1 9 0}\end{array}$ & $\begin{array}{c}\text { Landscapes } \\
n=193\end{array}$ & $\begin{array}{c}\text { Men } \\
\mathrm{n}=\mathbf{2 1 2}\end{array}$ \\
\hline \multirow{2}{*}{$\begin{array}{l}\text { Prime } \\
\text { Category }\end{array}$} & Landscapes & $0.91 * *(1.33)$ & $0.28 * *(0.90)$ & $2.06 * *(1.50)$ & $0.12 * *(0.44)$ \\
\hline & Men & $0.49 * *(1.18)$ & $0.99 * *(1.40)$ & $0.67 * *(1.23)$ & $0.51 * *(1.07)$ \\
\hline
\end{tabular}

Notes. All priming scores were significantly larger than zero with $p<.05$ (indicated by $*$ ), or $p<.001$ (indicated with **). The dependent measure is the difference between target ratings after positive primes and after negative primes.

The effect of conceptual overlap was a three-way interaction of prime category, target category and prime valence, $F(1,800)=202.31, p<.001, \eta_{\mathrm{p}}{ }^{2}=.20,90 \% C I[.16, .24]$. That effect reflected a stronger prime valence effect when the prime and the target belonged to the same category, than when they belonged to different categories. Specifically, when the target category was landscapes, the effect of prime valence was moderated by prime category, $F(1$, $400)=124.16, p<.001, \eta_{\mathrm{p}}{ }^{2}=.24,90 \% C I[.18, .29]$, reflecting a stronger prime valence effect when the prime category was landscapes, $F(1,400)=441.37, p<.001, \eta_{\mathrm{p}}{ }^{2}=.52$, than when the prime category was people, $F(1,400)=92.44, p<.001, \eta_{\mathrm{p}}^{2}=.19$. When the target category was people, the effect of prime valence was moderated by prime category, $F(1,400)=78.26, p<$ $.001, \eta_{\mathrm{p}}{ }^{2}=.16,90 \% C I[.06, .12]$, reflecting a stronger prime valence effect when the target 
category was people, $F(1,400)=146.02, p<.001, \eta_{p}{ }^{2}=.27$, than when the target category was landscapes, $F(1,400)=33.19, p<.001, \eta_{\mathrm{p}}{ }^{2}=.08$.

The three-way interaction of prime category, target category and prime valence was further moderated by target medium, $F(1,800)=9.93, p=.002, \eta_{\mathrm{p}}^{2}=.01$, reflecting a slightly larger overlap effect when the targets were photographic images, $F(1,403)=134.04, p<.001, \eta_{\mathrm{p}}{ }^{2}=$ .25 , than when the targets were sketches, $F(1,397)=70.47, p<.001, \eta_{\mathrm{p}}{ }^{2}=.15$.

\section{Discussion}

Experiment 2 replicated the results of Experiment 1 with both photographic and sketch targets. Participants were more likely to misattribute when the prime and the target were of the same category than when their categories differed. The effect was large even when the targets were sketches and the primes were photos, which increases the likelihood that it is the result of conceptual overlap rather than perceptual overlap. Although the effect was stronger when both primes and targets were photos, it is difficult to know whether the reason was that perceptual overlap (which caused perceptual confusion) also contributes to the priming effect in the AMP. It is reasonable to assume that live photos share conceptual features that they do not share with sketches. For example, sketches are often humorous and less serious than photographs. Consequently, a picture of a real person is more likely (than a sketch) to convey candid human emotions, which are more likely to be misattributed to a real human face than to a sketch.

In parallel to the effect of conceptual overlap, the results show that misattribution was considerably more likely when the targets were landscapes than people. Consequently, when the primes were people and the targets were photographic, misattribution was stronger when the targets were landscapes (the low conceptual overlap condition in that comparison) rather than people (Table 2). Although unrelated to the present research question, we speculate that the 
larger priming effect for landscape targets is a result of a greater room for interpretation. While there are relatively fixed criteria for judging the pleasantness of a face (e.g., a smile is always very important), the pleasantness of a scenery is more subjective and contextual. A dry field, for instance, can seem either lifeless and sad or charming and pastoral. Consequently, a face with average properties and a neutral facial expression would mostly get an average rating, but a dry field might get a very low rating when appearing after a dumping site and a very high rating after a beautiful scenery.

\section{Experiment 3}

We conducted this experiment as a conceptual replication of the previous experiments, using a different type of target stimuli: words. Participants judged the pleasantness of names rather than pictorial stimuli. We examined whether the effect of prime-target overlap replicates when the stimuli are semantic symbols rather than visual objects. That is a highly stringent test of the conceptual overlap hypothesis because people probably categorize word stimuli rather differently than image stimuli. People use different attributes when they judge photos and names. Even if the photo and the name refer to the same concept (e.g., a man), much of the content activated by the photo (e.g., kind eyes) would be less applicable when judging a name. Therefore, names would not seem a likely source of much of the mental content activated by photos. In other words, the potential for misattribution from a prime photo to a target word, even when the two overlap conceptually, is much lower than the potential for misattribution when both prime and target are photos. Although stringent, this test is advantageous in that perceptual confusion between images and words is very unlikely. 


\section{Method}

Participants. 663 participants completed the experiment, out of which 74 participants were excluded (using the same exclusion rule as in Experiments 1-2), and 589 entered the analysis $\left(68 \%\right.$, women, $\left.M_{\text {age }}=31.50, S D_{\text {age }}=13.36\right)$. This sample size yielded $93 \%$ power to detect a three-way interaction of prime category, target category, and prime valence, with an effect size of $\eta_{\mathrm{p}}^{2}=.02$.

Design. The design was a 2 (target category: names of landscapes, name of people; between participants) x 2 (prime category: landscapes, people; within participants) x 2 (prime valence: positive, negative; within participants).

Materials. The prime stimuli were images of positive and negative landscapes, women and men (mostly the same as in Experiments 1-2; taken from the DepositPhotos image pool, from online public domains, and from the IAPS). There were twelve men images, twelve women images and twelve landscape images (36 in total), half pleasant and half unpleasant. The targets were 24 male names, 24 female names, and 24 names of natural landmarks in the United States (e.g., Mississippi River).

Procedure. Participants completed four blocks of 24 trials. Each participant was randomly assigned to rate either names of people or names of landscapes (24 names, each name presented once in each block). Among participants who rated names of people, half were randomly assigned to rate male names and half female names. Participants who judged female names saw primes depicting either women or landscapes (all 12 women primes and 12 landscape primes appeared once in each block), and participants who judged male names saw primes depicting either men or landscapes (all 12 men primes and 12 landscape primes appeared once in each block). Participants who judged landscape names, saw primes depicting either women, men or 
landscapes ( 6 women primes and 6 men primes appeared in each block). Participants were instructed to judge how much they liked each of the names of women [men, landscapes], while trying to get a sense of whether they feel that a woman [man, landscape] with this name would be a pleasant person [sight].

\section{Results}

Table 3 shows the priming scores in each prime and target condition. The table shows that when targets were names of men, priming was clearly stronger when the primes were men compared to landscapes. When the targets were names of women, priming was somewhat stronger when the primes were women compared to landscapes. Finally, when the targets were names of landscapes, priming was slightly stronger when primes were landscapes compared to women and men.

We conducted a 2 (target category: names of landscapes, name of people) x 2 (prime category: landscapes, people) x 2 (prime valence: positive, negative) ANOVA on the average ratings. A main effect of prime valence, $F(1,587)=84.86, p<.001, \eta_{\mathrm{p}}{ }^{2}=.13$, reflected the expected priming effect. There was a main effect of target category, $F(1,587)=10.79, p=.001$, $\eta_{\mathrm{p}}{ }^{2}=.02$, reflecting more positive ratings of landscape names than people's names. There was also a main effect of prime category, $F(1,587)=29.16, p<.001, \eta_{\mathrm{p}}{ }^{2}=.05$, reflecting more positive ratings when primes were landscapes compared to people. A prime category by prime valence interaction, $F(1,587)=5.15, p=.024, \eta_{\mathrm{p}}{ }^{2}=.01$, reflected slightly larger priming after people primes compared to after landscape primes. A target category by prime category interaction, $F(1,587)=13.52, p<.001, \eta_{\mathrm{p}}^{2}=.02$, reflected more positive ratings after landscape compared to after people primes when targets were names of landscapes, $F(1,215)=33.72, p<$ $.001, \eta_{\mathrm{p}}{ }^{2}=.15$, but not when targets were names of people, $F(1,378)=1.98, p=.160, \eta_{\mathrm{p}}{ }^{2}=.01$. 
Table 3.

Experiment 3: Mean Priming Scores by Target Category and Prime Category (standard deviation in parentheses).

\begin{tabular}{lcccc}
\hline & & \multicolumn{3}{c}{ Target Category } \\
& Landscapes & Men & Women \\
& & $\mathbf{n}=\mathbf{2 1 6}$ & $\mathbf{n}=\mathbf{1 8 6}$ & $\mathbf{n = 1 8 7}$ \\
$\begin{array}{l}\text { Prime } \\
\text { Category }\end{array}$ & Landscapes & $0.40^{* *}(0.87)$ & $0.21 * *(0.85)$ & $0.20^{*}(0.87)$ \\
& Men & $0.35^{* *}(1.05)$ & $0.40^{* *}(0.96)$ & - \\
& Women & $0.37 * *(1.03)$ & - & $0.27 * *(0.83)$
\end{tabular}

Notes. All priming scores were significantly larger than zero with $p<.01$ (indicated by $*$ ), or $p<.001$ (indicated with **).

Most importantly, a three-way interaction of prime category, target category and prime valence, $F(1,587)=7.47, p=.006, \eta_{\mathrm{p}}^{2}=.01,90 \% C I[.00, .03]$, reflected a prime-target overlap effect. When the target category was people, the effect of prime valence was moderated by the prime category, $F(1,371)=10.33, p=.001, \eta_{\mathrm{p}}{ }^{2}=.03$, reflecting a larger effect of prime valence when the primes were people, $F(1,372)=52.33, p<.001, \eta_{\mathrm{p}}^{2}=.12$, compared to when the primes were landscapes, $F(1,361)=22.09, p<.001, \eta_{\mathrm{p}}^{2}=.06$. When the target category was landscapes, however, the effect of prime valence was not moderated by prime category, $F(1$, $215)=0.94, p=.443, \eta_{\mathrm{p}}^{2}<.01$.

\section{Discussion}

Experiment 3 replicated the results of Experiments 1 and 2 with word targets. Participants misattributed more when the image primes were of the same conceptual category as the word targets. This replication further confirms that conceptual (and not only perceptual) overlap 
increases misattribution. The small effect size for moderation by conceptual overlap in this experiment is probably a result of a considerably weaker manipulation of conceptual overlap. Because we used names as targets, there was a strong commonality between the landscape and people targets: both were names. To a certain degree, names might belong to a similar conceptual category, regardless of the object they represent. It is rather common for a place to be named after a person or a person after a place. Thus, names of landscapes and people might be particularly similar in the way they are commonly categorized. Further, the conceptual distance between names (the targets in this study) and visual objects (the primes) might be rather large, even between names and visual objects that typically go together (a landscape name and a landscape image or a person's name and a person's image). Despite this weakness, we still found statistically reliable evidence that a larger conceptual overlap between the primes and the targets increased misattribution.

\section{Experiment 4}

Although we used different stimuli in Experiments 1-3, the categories were always landscapes and people. To generalize our results beyond these categories, in Experiment 4, we manipulated prime and target categories with three new categories: dogs, flowers, and food.

\section{Method}

Participants. 899 participants completed the experiment, 60 were excluded (using the same exclusion rule as in the previous experiments), and 839 (93\%) entered the analysis (56\%, women, $\left.M_{\text {age }}=35.17, S D_{\text {age }}=14.54\right)$. The sample size achieved $96 \%$ power to detect the effect of conceptual overlap (a three-way interaction of prime category, target category, and prime valence), with an effect size of $\eta_{\mathrm{p}}{ }^{2}=.02$. 
Design. The design was 3 (target category: flowers, dogs, food; between participants) x 3 (prime category: flowers, dogs, food; within participants) x 2 (prime valence: positive, negative; within participants).

Materials. The prime stimuli were 54 photographic images of positive and negative flowers, dogs and food (nine of each valence-category combination; taken from the DepositPhotos image pool, from online public domains, and from the IAPS). The negative flowers were decaying, grey and thorny, negative food was stale, and the negative dogs looked either hostile or miserable. The positive flowers looked fresh and colorful, positive food was commonly desired meals such as hamburger and fries, and positive dogs were cute and friendly. As targets, each participant rated 36 sketch images that belonged to one of three categories: flowers, dogs or food (taken from the Depositphotos image pool).

Procedure. Participants completed three blocks of 36 trials, using the same procedure as the previous experiments. Each block showed the same 36 targets (all the targets in the set). We instructed participants to judge how pleasant or unpleasant the flower, dog, or food seemed to them. The total number of primes (54) was half of the total number of trials in the task (108). The primes were assigned randomly without replacement to each trial, so that each prime appeared twice during the task. Within each block, there were six trials for each of the six prime valence $\mathrm{X}$ prime category combinations, for a total of 18 trials for each combination throughout the task.

\section{Results}

Table 4 shows the priming score in each experimental condition. The table shows that, for each target category, priming was stronger when the primes belonged to the same category as the targets. We conducted a 3 (target category: flowers, dogs, food) x 3 (prime category: flowers, 
dogs, food) x 2 (prime valence: positive, negative) ANOVA on the average ratings. A main effect of prime valence, $F(1,836)=314.78, p<.001, \eta_{\mathrm{p}}{ }^{2}=.259$, reflected the expected priming effect. A main effect of target category, $F(2,836)=3.43, p=.033, \eta_{\mathrm{p}}{ }^{2}=.01$, reflected more positive evaluations of food $(M=4.753, S D=0.765)$ compared to flowers $(M=4.603, S D=$ $0.760)$ and $\operatorname{dogs}(M=4.620, S D=0.725)$. A main effect of prime category, $F(2,1672)=10.24, p$ $<.001, \eta_{\mathrm{p}}{ }^{2}=.01$, reflected more negative evaluations of the targets when primes were dogs $(M=$ 4.621, $S D=0.774)$ compared to flowers $(M=4.675, S D=0.800)$ and food $(M=4.689, S D=$ 0.809).

The effect of conceptual overlap was a three-way interaction of prime category, target category and prime valence, $F(4,1672)=105.02, p<.001, \eta_{\mathrm{p}}^{2}=.194,90 \% C I[.172, .227]$. This effect reflected a stronger prime valence effect when the prime and the target belonged to the same category, than when they belonged to different categories. When the target category was dogs, the effect of prime valence was moderated by the prime category, $F(2,570)=142.06, p<$ $.001, \eta_{\mathrm{p}}{ }^{2}=.33$, reflecting a larger effect of prime valence when the primes were dogs, $F(1,285)$ $=173.90, p<.001, \eta_{\mathrm{p}}^{2}=.38$, compared to when the primes were flowers, $F(1,285)=13.21, p<$ $.001, \eta_{\mathrm{p}}{ }^{2}=.04$, and when the primes were foods, $F(1,285)=5.66, p<.001, \eta_{\mathrm{p}}{ }^{2}=.02$. When the target category was flowers, the effect of prime valence was moderated by the prime category, $F(2,512)=39.01, p<.001, \eta_{\mathrm{p}}^{2}=.13$, reflecting a larger effect of prime valence when the primes were flowers, $F(1,256)=118.13, p<.001, \eta_{\mathrm{p}}{ }^{2}=.32$, compared to when the primes were dogs, $F(1,256)=43.32, p<.001, \eta_{\mathrm{p}}^{2}=.14$, and when the primes were foods, $F(1,256)=30.88, p<$ $.001, \eta_{\mathrm{p}}{ }^{2}=.11$. Finally, when the target category was food, the effect of prime valence was moderated by the prime category, $F(2,590)=21.68, p<.001, \eta_{\mathrm{p}}{ }^{2}=.07$, reflecting a larger effect of prime valence when the primes were food, $F(1,295)=81.45, p<.001, \eta_{\mathrm{p}}{ }^{2}=.22$, compared to 
when the primes were dogs, $F(1,295)=24.73, p<.001, \eta_{\mathrm{p}}{ }^{2}=.08$, and when the primes were flowers, $F(1,295)=26.33, p<.001, \eta_{\mathrm{p}}{ }^{2}=.08$.

\section{Table 4.}

Experiment 4: Mean Priming Scores by Target Category and Prime Category (standard deviation in parentheses).

\begin{tabular}{lcccc}
\hline & \multicolumn{3}{c}{ Target Category } \\
& & $\begin{array}{c}\text { Dogs } \\
\mathbf{n}=\mathbf{2 8 6}\end{array}$ & $\begin{array}{c}\text { Flowers } \\
\mathbf{n = 2 5 7}\end{array}$ & $\begin{array}{c}\text { Food } \\
\mathbf{n}=\mathbf{2 9 6}\end{array}$ \\
$\begin{array}{l}\text { Prime } \\
\text { Category }\end{array}$ & Dogs & $1.24 *(1.56)$ & $0.40 * *(0.98)$ & $0.24 * *(0.83)$ \\
& Flowers & $0.10^{*}(0.47)$ & $0.77 * *(1.13)$ & $0.24 * *(0.81)$ \\
& Food & $0.06 *(0.48)$ & $0.25^{* *}(0.71)$ & $0.56^{* *}(1.07)$
\end{tabular}

Notes. All priming scores were significantly larger than zero with $p<.05$ (indicated by *), or $p<.01$ (indicated with $* *$ ). The dependent measure is the difference between target ratings after positive primes and after negative primes.

\section{Discussion}

Experiment 4 replicated the results of Experiments 1-3 with a more diverse set of prime and target categories. For each target they judged - either food, flowers or dogs-participants were more likely to misattribute when the prime and the target were of the same category than when their categories differed. Dogs, flowers and food differ in the internal experiences that they trigger. Whereas a positive dog might make participants think of something cute and loyal, positive flowers and food are neither of those things. The main commonality between food, dogs and flowers is that they all can be judged positively or negatively. So, a negative image of food triggers a generally negative experience that can be attributed to almost any object. Indeed, we 
consistently see priming effects in the low conceptual overlap conditions. Importantly, however, a higher match in conceptual content increases the chance that attribution will occur.

\section{General Discussion}

In four experiments, we found that primes had a larger effect on judgment of target stimuli when the primes and the targets belonged to the same category than when they belonged to different categories. This result suggests that misattribution is stronger when the object that activated the internal experience and the object to which the experience is attributed share a larger conceptual overlap. We replicated this finding when the targets were words, photos and sketches, and with a diverse set of prime and target categories.

Our findings are compatible with the assumption that people utilize the conceptual overlap between the internal experience and the target when making attributions. People attribute rich internal experiences rather than isolated properties such as valence or arousal. The internal experience carries specific semantic information about the object that caused it, and that information guides the attribution process.

If misattribution is sensitive to the specific properties of primes and targets, does that mean that people are less or more confused about the source of their experiences than thought before? Our results show that misattribution occurs even when conceptual overlap is low. That is consistent with previous research that used, perhaps exclusively, primes and targets of low conceptual overlap. However, in real life, conceptual overlap is probably not always as small as in most previous research on misattribution. Because our results show that high overlap increases misattribution, it might be that in real life misattribution is even stronger and more prevalent than previous results suggest. 
Our results might challenge old views about the nature of attribution errors. Past findings on misattribution seem to show that people can misattribute internal experiences from any prime to any target to a similar extent. For instance, White et al. (1981) found that participants misattributed feelings of arousal to romantic attraction to a similar extent when the arousing prime was a comedy audio segment and when it was an audio segment about mutilations. This finding seems to indicate that any arousing prime would cause similar misattribution. However, notice that both humor and mutilations are conceptually distant from romance and attraction. Our results suggest that a prime with a high conceptual overlap (e.g., an audio segment about famous attractive people) would have caused more misattribution to attraction than both comedy and mutilations.

Importantly, however, generalizing our results to conceptual overlap in attribution in general warrants caution. It is possible that the influence of conceptual overlap on misattribution is limited to the conditions in the AMP: when the primes are clearly valenced, the targets are relatively neutral, primes and targets appear briefly and in close succession, and multiple targets are judged in a relatively short period of time. More specifically, it is a reasonable possibility that the similarity between the prime and the target would not be as consequential for attribution when the prime's salience is reduced and the target appears only after a delay, as were the standard conditions in past research (Cantor et al., 1975; Reisenzein \& Gattinger, 1982; White et al., 1981). Perhaps sometime after a non-salient priming event, the residual internal experience no longer carries rich semantic information about the object that caused it, and could thus be attributed similarly to conceptually similar and dissimilar objects. 


\section{Correction Processes}

An important previous finding about misattribution in the AMP is that participants are generally immune to warnings about the primes' influence on their judgments (Payne et al., 2005). According to Payne et al. (2005), warnings in the AMP are futile because participants fail to identify that the primes triggered the internal experience. The current findings are compatible with the notion that resources for reducing misattribution are not recruited or are ineffective in reducing misattribution in the AMP. Although the prime's similarity to the target is likely to increase the suspicion that the prime would bias judgment, and warnings about this possibility appeared throughout the task, participants did not correct for it. On the contrary, they misattributed more when the possibility of bias was more apparent.

According to Wilson and Brekke (1994), warnings about bias are effective when (1) people believe the warning, (2) are motivated to avoid bias, and (3) are able to avoid bias. By making the possibility of bias more apparent, high conceptual overlap increased the likelihood that participants would believe the warning. One possible explanation for our results is that, although participants believed the warning, they were unmotivated to avoid bias. However, this possibility is unlikely because research suggests that people are generally motivated to judge accurately (Schul, 1993; Wegener \& Petty, 1997; Wilson \& Brekke, 1994), unless inaccurate judgment serves other motives (Kunda, 1990). A more plausible account might be that participants were unable to avoid misattribution. People seem to have relatively poor skills in attributing their internal experiences under time pressure (Greifeneder \& Bless, 2007; Siemer \& Reisenzein, 1998). They might need to deliberate and invest more cognitive resources in their judgment to enable correct attribution. Although responses in the AMP are not limited by time, the presentation of the stimuli is very brief, which might discourage people from long deliberation 
based on the memory of briefly presented stimuli. Further, because the AMP involves dozens of judgments, people might opt out from recruiting deliberate and effortful processes.

\section{Implications for the AMP}

As discussed earlier, one limitation of the present research is that it is possible that our findings are limited to the settings captured by the AMP. By the same token, our results could be highly informative for understanding the AMP as an indirect measure of social cognition. In most versions of the AMP, the conceptual overlap between primes and targets is not high — the targets are Chinese letters and the primes are neither Chinese nor letters. Because the measurement of automatic evaluation with the AMP depends on the extent to which participants misattribute to the target their evaluation of the prime, higher conceptual overlap would increase the AMP's sensitivity. Consequently, the AMP would be able to measure weaker evaluations and distinguish between subtler differences in evaluation.

Further, when using the AMP to measure evaluation of multiple objects, it would be important to keep an equal conceptual overlap between all prime categories and the targets of judgment. Otherwise, differences in attitude extremity in the AMP might reflect differences in prime-target conceptual overlap, rather than differences in the actual extremity of the attitudes toward the prime object.

\section{Alternative Accounts}

Could the reason for the present finding be that conceptual overlap increased intentional rating of the primes? Previous research found that people who show stronger priming effects are more likely to report intentional rating of the primes rather than the targets (Bar-Anan \& Nosek, 2012). These reports could reflect actual rating of the primes or confabulated memory of such 
intentional rating (Bar-Anan \& Nosek, 2012; Payne et al., 2013). However, no research to date has shown that participants indeed rate the primes intentionally. In fact, some research found priming effects in the AMP that cannot be explained by intentional rating of primes (Gawronski $\&$ Ye, 2015; Payne, Govorun, \& Arbuckle, 2008). Further, the present research used a modified version of the AMP to decrease the risk of eliciting motivation to defy task instructions: Instead of judging highly abstract and dull symbols as pleasant or unpleasant, the present task presented less monotonous target sets, and provided a more reasonable 7-point judgment scale. Finally, it is unclear why people would prefer to rate the primes when they are similar to the targets, rather than when they include unique content that the targets do not.

Another alternative for our interpretation of the present results is that, rather than intentionally defying task instructions, participants rated the primes because they misread the instructions or were confused during the task. According to that alternative account, the reason for stronger priming when primes and targets had a higher conceptual overlap is that the task is more confusing when conceptual overlap is high (because primes and targets are more similar). We attempted to prevent confusion by repeating the instructions and emphasizing that participants should rate the stimulus in the orange frame (only targets appeared in the orange frame). More importantly, in Experiments 1 and 3 we used live images as primes and sketches as targets. When participants are explicitly told that they should rate the sketches in the orange frame, it seems unlikely that they would accidentally rate the live images. In Experiment 2, the targets were words and the primes were live photos. The clear instructions to rate the words similarly reduce the threat of confusion regarding what stimulus should be rated on each trial.

In closing, we note that the study of misattribution is a window into attribution processes in general. The current findings offer an important addition to past work that focused mainly on 
the effect of primes that trigger general valence (e.g., the standard AMP procedure) or general arousal (Reisenzein \& Gattinger, 1982; Schachter \& Singer, 1962; White et al., 1981), and ignored the relation between the category of the primes and the category of the targets. Our results point at the major role played by specific content of the internal experience that the prime triggers. The pleasantness of a scenery is not the same as the pleasantness of a person, and would not be similarly misattributed. People are specific in the attributions they make: they attribute scenery pleasantness to scenery and person pleasantness to a person. Consequently, a

politician's efforts to be likable would yield better results if she attempted to be seen with likable objects that are similar to her (e.g., people, politicians, women) rather than likable dissimilar objects (e.g., babies, pets).

\section{Open Practices}

The experiments presented in this paper earned Open Data and Open Materials badges for transparent practices. Materials and data for all experiments are available at: https://osf.io/cgydx/. 


\section{References}

Aarts, H., \& Dijksterhuis, A. (2002). Category activation effects in judgment and behaviour: The moderating role of perceived comparability. British Journal of Social Psychology, 41, 123138.

Bar-Anan, Y., \& Nosek, B. A. (2012). Reporting intentional rating of the primes predicts priming effects in the affective misattribution procedure. Personality and Social Psychology Bulletin, 38, 1194-1208.

Barrett, L. F. (2006). Solving the emotion paradox: Categorization and the experience of emotion. Personality and Social Psychology Review, 10, 20-46.

Bem, D. J. (1972). Self-perception theory. In L. Berkowitz (Ed.), Advances in experimental social psychology (Vol. 6, pp. 2-62). New York: Academic Press

Blaison, C., Imhoff, R., Huhnel, I., Hess, U., \& Banse, R. (2012). The affect misattribution procedure: Hot or not? Emotion, 12, 403-412.

Bless, H., \& Schwarz, N. (2010). Mental construal and the emergence of assimilation and contrast effects: The inclusion/exclusion model. Advances in Experimental Social Psychology, 42, 319 -373. doi:10.1016/ S0065-2601(10)42006-7

Bradley, M. M., \& Lang, P. J. (2007). The International Affective Picture System (IAPS) in the study of emotion and attention. In J. A. Coan \& J. J. B. Allen (Eds.), Handbook of Emotion Elicitation and Assessment (pp. 29 - 46). Oxford, England: Oxford University Press.

Bräscher A, Raymaekers K, van den Bergh O, Witthöft M. (2017). Are media reports able to cause somatic symptoms attributed to WiFi radiation? An experimental test of the negative expectation hypothesis. Environmental Reports, 156, 265-71. 
Cantor, J. R., Zillmann, D., \& Bryant, J. (1975). Enhancement of experienced sexual arousal in response to erotic stimuli through misattribution of unrelated residual excitation. Journal of Personality and Social Psychology, 32, 69-75.

De Houwer, J., \& Smith, C. T. (2013). Go With Your Gut!. Social Psychology, 44, 299-302.

Deutsch, R., \& Gawronski, B. (2009). When the method makes a difference: Antagonistic effects on "automatic evaluations" as a function of task characteristics of the measure. Journal of Experimental Social Psychology, 45, 101-114.

Dutton, D. G., \& Aron, A. P. (1974). Some evidence for heightened sexual attraction under conditions of high anxiety. Journal of Personality and Social Psychology, 30, 510-517.

Ecker, Y. \& Bar-Anan, Y. (2018). Applicability Increases the Effect of Misattribution on Judgment. Cognition \& Emotion, 1-13.

Faul, F., Erdfelder, E., Lang, A. G., \& Buchner, A. (2007). G* Power 3: A flexible statistical power analysis program for the social, behavioral, and biomedical sciences. Behavior Research Methods, 39, 175-191.

Gawronski, B., Cunningham, W. A., LeBel, E. P., \& Deutsch, R. (2010). Attentional influences on affective priming: Does categorisation influence spontaneous evaluations of multiply categorizable objects?. Cognition and Emotion, 24, 1008-1025.

Gawronski, B., \& Ye, Y. (2014). What drives priming effects in the affect misattribution procedure?. Personality and Social Psychology Bulletin, 40, 3-15.

Gawronski, B., \& Ye, Y. (2015). Prevention of intention invention in the affect misattribution procedure. Social Psychological and Personality Science, 6, 101-108. 
Greifeneder, R., \& Bless, H. (2007). Relying on accessible content versus accessibility experiences: The case of processing capacity. Social Cognition, 25, 853-881.

Hofmann, W., \& Baumert, A. (2010). Immediate affect as a basis for intuitive moral judgment: An adaptation of the affect misattribution procedure. Cognition \& Emotion, 24, 522-535

Imhoff, R., Schmidt, A. F., Bernhardt, J., Dierksmeier, A., \& Banse, R. (2011). An inkblot for sexual preference: A semantic variant of the Affect Misattribution Procedure. Cognition and Emotion, 25, 676-690.

Laird, J. (1974). Self-attribution of emotion: The effects of expressive behavior on the quality of emotional experience. Journal of Personality and Social Psychology, 29, 475-486.

LeBoeuf, R. A., \& Estes, Z. (2004). “Fortunately, I'm no Einstein”: Comparison relevance as a determinant of behavioral assimilation and contrast. Social Cognition, 22, 607-636.

Minear, M., \& Park, D. C. (2004). A lifespan database of adult facial stimuli. Behavior Research Methods, Instruments, \& Computers, 36, 630-633.

Moskowitz, G. B., \& Skurnik, I. W. (1999). Contrast effects as determined by the type of prime: Trait versus exemplar primes initiate processing strategies that differ in how accessible constructs are used. Journal of Personality and Social Psychology, 76, 911-927.

Murphy, S. T., \& Zajonc, R. B. (1993). Affect, cognition, and awareness: affective priming with optimal and suboptimal stimulus exposures. Journal of Personality and Social Psychology, 64, 723-739.

Nosek, B. A. (2005). Moderators of the relationship between implicit and explicit evaluation. Journal of Experimental Psychology: General, 134, 565-584. 
Nosek, B. A., \& Banaji, M. R. (2001). The go/no-go association task. Social Cognition, 19, 625666.

Oikawa, M., Aarts, H., \& Oikawa, H. (2011). There is a fire burning in my heart: The role of causal attribution in affect transfer. Cognition and Emotion, 25, 156-163.

Payne, B. K. (2001). Prejudice and perception: the role of automatic and controlled processes in misperceiving a weapon. Journal of Personality and Social Psychology, 81, 181.

Payne, B. K., Brown-Iannuzzi, J., Burkley, M., Arbuckle, N. L., Cooley, E., Cameron, C. D., \& Lundberg, K. B. (2013). Intention invention and the affect misattribution procedure: Reply to Bar-Anan and Nosek (2012). Personality and Social Psychology Bulletin, 39, 375-386.

Payne, B. K., Burkley, M. A., \& Stokes, M. B. (2008). Why do implicit and explicit attitude tests diverge? The role of structural fit. Journal of personality and social psychology, 94, 16-31.

Payne, B. K., Cheng, C. M., Govorun, O., \& Stewart, B. D. (2005). An inkblot for attitudes: affect misattribution as implicit measurement. Journal of Personality and Social Psychology, 89, 277.

Payne, B. K., Govorun, O., \& Arbuckle, N. L. (2008). Automatic attitudes and alcohol: Does implicit liking predict drinking?. Cognition \& Emotion, 22, 238-271.

Payne, B. K., Hall, D. L., Cameron, C. D., \& Bishara, A. J. (2010). A process model of affect misattribution. Personality and Social Psychology Bulletin, 36, 1397-1408.

Quine, W. V. O. (1960). Word and object. Cambridge, England: Cambridge University Press. Reisenzein, R., \& Gattinger, E. (1982). Salience of arousal as a mediator of misattribution of transferred excitation. Motivation and Emotion, 6, 315-328. 
Russell, J. A. (2003). Core affect and the psychological construction of emotion. Psychological Review, 110, 145-172.

Ruys, K. I., Aarts, H., Papies, E. K., Oikawa, M., \& Oikawa, H. (2012). Perceiving an exclusive cause of affect prevents misattribution. Consciousness and Cognition, 21, 1009-1015.

Schachter, S., \& Singer, J. (1962). Cognitive, social, and physiological determinants of emotional state. Psychological Review, 69, 379.

Schnall, S., Abrahamson, A., \& Laird, J. D. (2002). Premenstrual syndrome and misattribution: A self-perception, individual differences perspective. Basic and Applied Social Psychology, 24, 215-228.

Schwarz, N., \& Clore, G. L. (1983). Mood, misattribution, and judgments of well-being: Informative and directive functions of affective states. Journal of Personality and Social Psychology, 45, 513-523.

Siemer, M., \& Reisenzein, R. (1998). Effects of mood on evaluative judgements: Influence of reduced processing capacity and mood salience. Cognition \& Emotion, 12, 783-805.

Treisman, A., \& Schmidt, H. (1982). Illusory conjunctions in the perception of objects. Cognitive Psychology, 14, 107-141.

White, G. L., Fishbein, S., \& Rutsein, J. (1981). Passionate love and the misattribution of arousal. Journal of Personality and Social Psychology, 41, 56-62.

Zhou, H., \& Fishbach, A. (2016). The Pitfall of Experimenting on the Web: How Unattended Selective Attrition Leads to Surprising (Yet False) Research Conclusions. Journal of Personality and Social Psychology. Advance online publication. http://dx.doi.org/10.1037/ pspa0000056 
Zlotnick, E., Dzikiewicz, A. J., \& Bar-Anan, Y. (2015). Minno.js (Version 0.3) [Computer software].

Wegener, D. T., \& Petty, R. E. (1995). Flexible correction processes in social judgment: The role of naive theories in corrections for perceived bias. Journal of personality and social psychology, 68, 36-51.

Wilson, T. D., \& Brekke, N. (1994). Mental contamination and mental correction: unwanted influences on judgments and evaluations. Psychological bulletin, 116, 117-142. 


\section{Supplementary Material}

\section{Correlations between Measures in Experiments 1-4}

Table S1. Experiment 1: The correlation between average rating after positive primes and average rating after negative primes, within each of the four between-participants conditions.

\begin{tabular}{|c|c|c|c|}
\hline & & \multicolumn{2}{|c|}{ Target type } \\
\hline & & People & Scenery \\
\hline \multirow[t]{2}{*}{ Prime type } & People & .469 & .477 \\
\hline & Scenery & .592 & .266 \\
\hline
\end{tabular}

Notes. Prime types and target types were manipulated between participants. Within each of these between-participants conditions, there were two repeated measures: average rating after positive primes and average rating after negative primes. The table presents correlation between these repeated measures for each of the between-participants conditions. All correlation scores were significantly larger than zero with $p<.001$. 
Table S2. Experiment 2: Correlations between repeated measures (average rating after each prime type and prime valence) in each level of the between-participants variable (target type).

\begin{tabular}{|c|c|c|c|c|c|}
\hline & & \multicolumn{4}{|c|}{ Photographic scenery targets: } \\
\hline & & \multicolumn{2}{|c|}{ Scenery primes } & \multicolumn{2}{|c|}{ People primes } \\
\hline & & Positive & Negative & Positive & Negative \\
\hline \multirow{2}{*}{$\begin{array}{l}\text { Scenery } \\
\text { primes }\end{array}$} & Positive & - & & & \\
\hline & Negative & $-.161 *$ & - & & \\
\hline \multirow{3}{*}{$\begin{array}{l}\text { People } \\
\text { primes }\end{array}$} & Positive & $.437 * *$ & $.449 * *$ & - & \\
\hline & Negative & 0.104 & $.227 * *$ & 0.096 & - \\
\hline & & \multicolumn{4}{|c|}{ Photographic people targets: } \\
\hline \multirow{2}{*}{$\begin{array}{l}\text { Scenery } \\
\text { primes }\end{array}$} & Positive & - & & & \\
\hline & Negative & $.762 * *$ & - & & \\
\hline \multirow{5}{*}{$\begin{array}{l}\text { People } \\
\text { primes }\end{array}$} & Positive & $.53 * *$ & $.489 * *$ & - & \\
\hline & Negative & $.637 * *$ & $.671 * *$ & .080 & - \\
\hline & & \multicolumn{4}{|c|}{ Sketch scenery targets: } \\
\hline & & \multicolumn{2}{|c|}{ Scenery primes } & \multicolumn{2}{|c|}{ People primes } \\
\hline & & Positive & Negative & Positive & Negative \\
\hline \multirow{2}{*}{$\begin{array}{l}\text { Scenery } \\
\text { primes }\end{array}$} & Positive & - & & & \\
\hline & Negative & $.224 * *$ & - & & \\
\hline \multirow{3}{*}{$\begin{array}{l}\text { People } \\
\text { primes }\end{array}$} & Positive & $.379 * *$ & $.238 * *$ & - & \\
\hline & Negative & 0.091 & $.629 * *$ & $-.211 *$ & - \\
\hline & & \multicolumn{4}{|c|}{ Sketch people targets: } \\
\hline
\end{tabular}




\begin{tabular}{|c|c|c|c|c|c|}
\hline \multirow{2}{*}{$\begin{array}{l}\text { Scenery } \\
\text { primes }\end{array}$} & Positive & - & & & \\
\hline & Negative & .083 & - & & \\
\hline \multirow{2}{*}{$\begin{array}{l}\text { People } \\
\text { primes }\end{array}$} & Positive & $.510 * *$ & $.588 * *$ & - & \\
\hline & Negative & -.078 & $.633 * *$ & $.295^{* *}$ & - \\
\hline
\end{tabular}

Notes. $*$ indicates that the priming score is significant with $p<.05 ; * *$ indicates that the priming score is significant with $p<.001$. 
Table S3. Experiment 3: Correlations between repeated measures (average rating after each prime type and prime valence) in each level of the between-participants variable (target type).

Scenery targets:

\begin{tabular}{|c|c|c|c|c|c|}
\hline & & \multicolumn{2}{|c|}{ Scenery primes } & \multicolumn{2}{|c|}{ People primes } \\
\hline & & Positive & Negative & Positive & Negative \\
\hline \multirow{2}{*}{$\begin{array}{l}\text { Scenery } \\
\text { primes }\end{array}$} & Positive & - & & & \\
\hline & Negative & $.299 * *$ & - & & \\
\hline \multirow{3}{*}{$\begin{array}{l}\text { People } \\
\text { primes }\end{array}$} & Positive & $.686 * *$ & $.291 * *$ & - & \\
\hline & Negative & .083 & $.788 * *$ & $.166^{*}$ & - \\
\hline & & \multicolumn{4}{|c|}{ People targets: } \\
\hline \multirow{2}{*}{$\begin{array}{l}\text { Scenery } \\
\text { primes }\end{array}$} & Positive & - & & & \\
\hline & Negative & $.613 * *$ & - & & \\
\hline \multirow{2}{*}{$\begin{array}{l}\text { People } \\
\text { primes }\end{array}$} & Positive & $.822 * *$ & $.662 * *$ & - & \\
\hline & Negative & $.521 * *$ & $.851 * *$ & $.591 * *$ & 一 \\
\hline
\end{tabular}

Notes. $*$ indicates that the priming score is significant with $p<.01$; ** indicates that the priming score is significant with $p<.001$. 
Table S4. Experiment 4: Correlations between repeated measures (average rating after each prime type and prime valence) in each level of the between-participants variable (target type).

\begin{tabular}{|c|c|c|c|c|c|c|c|}
\hline & & \multicolumn{6}{|c|}{ Food targets: } \\
\hline & & \multicolumn{2}{|c|}{ Food primes } & \multicolumn{2}{|c|}{ Dog primes } & \multicolumn{2}{|c|}{ Flower primes } \\
\hline & & Positive & Negative & Positive & Negative & Positive & Negative \\
\hline \multirow{2}{*}{$\begin{array}{l}\text { Food } \\
\text { primes }\end{array}$} & Positive & - & & & & & \\
\hline & Negative & $.356^{*}$ & - & & & & \\
\hline \multirow{2}{*}{$\begin{array}{l}\text { Dog } \\
\text { primes }\end{array}$} & Positive & $.569 *$ & $.504 *$ & - & & & \\
\hline & Negative & $.501 *$ & $.729 *$ & $.611 *$ & - & & \\
\hline \multirow{3}{*}{$\begin{array}{l}\text { Flower } \\
\text { primes }\end{array}$} & Positive & $.591 *$ & $.501 *$ & $.879 *$ & $.559 *$ & - & \\
\hline & Negative & $.493 *$ & $.746^{*}$ & $.621 *$ & $.863 *$ & $.599 *$ & - \\
\hline & & \multicolumn{6}{|c|}{ Dog targets: } \\
\hline \multirow{2}{*}{$\begin{array}{l}\text { Food } \\
\text { primes }\end{array}$} & Positive & - & & & & & \\
\hline & Negative & $.839 *$ & - & & & & \\
\hline \multirow{2}{*}{$\begin{array}{l}\text { Dog } \\
\text { primes }\end{array}$} & Positive & $.638 *$ & $.574 *$ & - & & & \\
\hline & Negative & $.402 *$ & $.398 *$ & -.158 & - & & \\
\hline \multirow{3}{*}{$\begin{array}{l}\text { Flower } \\
\text { primes }\end{array}$} & Positive & $.923 *$ & $.844 *$ & $.644 *$ & $.363 *$ & - & \\
\hline & Negative & $.850 *$ & $.898 *$ & $.566^{*}$ & $.451 *$ & $.841 *$ & - \\
\hline & & \multicolumn{6}{|c|}{ Flower targets: } \\
\hline
\end{tabular}

Positive 


\begin{tabular}{|c|c|c|c|c|c|c|c|}
\hline $\begin{array}{l}\text { Food } \\
\text { primes }\end{array}$ & Negative & $.648 *$ & - & & & & \\
\hline \multirow{2}{*}{$\begin{array}{l}\text { Dog } \\
\text { primes }\end{array}$} & Positive & $.829 *$ & $.530 *$ & - & & & \\
\hline & Negative & $.619 *$ & $.842 *$ & $.432 *$ & - & & \\
\hline \multirow{2}{*}{$\begin{array}{l}\text { Flower } \\
\text { primes }\end{array}$} & Positive & $.666^{*}$ & $.412 *$ & $.723 *$ & $.353 *$ & - & \\
\hline & Negative & $.624 *$ & $.823 *$ & $.517 *$ & $.722 *$ & $.316^{*}$ & - \\
\hline
\end{tabular}

Notes. All correlation scores were significantly larger than zero with $p<.01 . *$ indicates that the priming score is significant with $p<.001$. 


\section{A comparison of continuous and binary versions of the AMP}

In a separate research project, we conducted three studies that compared a continuous and a binary version of the AMP. All materials pertaining to this project are available on the OSF (https://osf.io/g57nh/). In Study 1, the prime stimuli were eight negative and eight positive images (from the International Affective Picture System, IAPS; Bradley \& Lang, 2007), eight positive words, (Paradise, Pleasure, Cheer, Friend, Splendid, Love, Smile, Enjoy), and eight negative words (Bomb, Abuse, Sadness, Pain, Poison, Grief, Ugly, Stink). In Studies 2-3, primes were seven positive and seven negative IAPS images (no word primes). The targets were 72 Chinese symbols (Payne et al., 2005). The judgment category labels were dichotomous (Pleasant and Unpleasant) for half of the participants, and continuous (1-Very unpleasant, 2 - Moderately unpleasant, 3 - Slightly unpleasant, 4- Slightly pleasant, 5 - moderately pleasant, and 6 - Very pleasant) for the other half. In Studies 2-3, judgments in the continuous scale AMP were on a 7point scale (we added a neutral category). As shown in Table S5, the priming effects of both AMP versions were of similar size, and so was the internal consistency (computed from the three blocks of each task). 
Table S5. Comparing priming scores and latencies in continuous and binary versions of the AMP.

\begin{tabular}{lccc} 
& Binary AMP & Continuous AMP \\
\hline Study 1 & Mean (SD) & $0.24(0.32)$ & $0.71(1.08)$ \\
Priming & $\boldsymbol{d}$ & 0.75 & 0.66 \\
& $\boldsymbol{\alpha}$ & .89 & .91 \\
Latency & Mean (SD) & $1,075.45(378.07)$ & $1,633.741(721.57)$
\end{tabular}

\section{Study 2}

Mean (SD) $\quad 0.20(0.30) \quad 0.86(1.28)$

$\begin{array}{llll}\text { Priming } & \boldsymbol{d} & 0.67 & 0.67 \\ & \boldsymbol{\alpha} & .89 & .94\end{array}$

\begin{tabular}{|c|c|c|c|}
\hline Latency & Mean (SD) & $995.30(398.364)$ & $1,606.92(701.08)$ \\
\hline \multicolumn{4}{|l|}{ Study 3} \\
\hline & Mean (SD) & $0.22(0.34)$ & $0.72(1.26)$ \\
\hline \multirow[t]{2}{*}{ Priming } & $d$ & 0.65 & 0.57 \\
\hline & $\alpha$ & .90 & .93 \\
\hline Latency & Mean (SD) & $1,104.48(550.48)$ & $1,771.30(1,118.60)$ \\
\hline
\end{tabular}

Notes. All priming scores were significantly larger than zero, $p<.0001 . \alpha$ indicates the measure's internal consistency (Cronbach's Alpha). $d$ indicated the priming effect size (Cohen's d).

We also examined the number of keys that participants used during the continuous AMP, to see whether participants took advantage of the larger response set afforded by the continuous 
scale. In Study 1, we found that, on average, participants used 4.96 keys out of the 6 possible keys $(\mathrm{SD}=1.31)$. The average number of keys used decreased to $4.079(\mathrm{SD}=1.32)$ when including only key used more than five times throughout the AMP. In Study 2, participants used and average of 5.64 keys out of $7(\mathrm{SD}=1.35)$, and 4.44 keys $(\mathrm{SD}=1.45)$ more than 5 times. In Study 2, participants used an average of 5.26 keys out of $7(\mathrm{SD}=1.52)$, and 4.03 keys $(\mathrm{SD}=$ 1.46) more than 5 times throughout the AMP.

Experiments 1-4: Mean rating (and standard deviation) by experimental condition

Table S6.

Experiment 1: Mean Rating Scores by Target Category, Prime Category, and Prime Valence (standard deviation in parentheses).

\begin{tabular}{|c|c|c|c|c|}
\hline & & & \multicolumn{2}{|c|}{ Target Category } \\
\hline & & & Landscapes & People \\
\hline \multirow{5}{*}{$\begin{array}{l}\text { Prime } \\
\text { Category }\end{array}$} & & $\mathrm{PP}$ & $4.64(0.85)$ & $3.96(0.74)$ \\
\hline & & $\mathrm{NP}$ & $4.08(0.85)$ & $3.84(0.74)$ \\
\hline & \multirow{3}{*}{ People } & PP & $4.55(0.82)$ & $3.93(0.74)$ \\
\hline & & & & \\
\hline & & NP & $4.29(0.91)$ & $3.82(0.74)$ \\
\hline
\end{tabular}

Notes. $\mathrm{PP}=$ positive prime; $\mathrm{NP}=$ negative prime. 
Table S7.

Experiment 2: Mean Rating Scores by Target Category, Prime Category, Prime Valence, and Target Medium (standard deviation in parentheses).

\begin{tabular}{|c|c|c|c|c|c|c|}
\hline & & & Sketch T & 'argets & Photogra] & hic Targets \\
\hline & & & \multicolumn{4}{|c|}{ Target Category } \\
\hline & & & Landscapes & Men & $\begin{array}{c}\text { Landscap } \\
\text { es }\end{array}$ & Men \\
\hline \multirow{4}{*}{$\begin{array}{l}\text { Prime } \\
\text { Category }\end{array}$} & & $\mathrm{PP}$ & $5.25(0.97)$ & $4.35(0.76)$ & $5.77(0.91)$ & $3.98(0.69)$ \\
\hline & Landscapes & $\mathrm{NP}$ & $4.34(0.10)$ & $4.07(0.66)$ & $3.71(1.02)$ & $3.86(0.68)$ \\
\hline & & $\mathrm{PP}$ & $4.77(0.87)$ & $4.56(0.85)$ & $4.75(0.80)$ & $4.08(0.79)$ \\
\hline & Men & NP & $4.29(1.14)$ & $3.58(0.92)$ & $4.08(1.01)$ & $3.58(0.79)$ \\
\hline
\end{tabular}

Notes. $\mathrm{PP}=$ positive prime $; \mathrm{NP}=$ negative prime. 
Table S8.

Experiment 3: Mean Rating Scores by Target Category, Prime Category and Prime Valence (standard deviation in parentheses).

\begin{tabular}{|c|c|c|c|c|c|}
\hline & & & \multicolumn{3}{|c|}{ Target Category } \\
\hline & & & Landscapes & Men & Women \\
\hline \multirow{3}{*}{\multicolumn{2}{|c|}{ Landscapes }} & $\mathrm{PP}$ & $4.77(0.79)$ & 4.35 (1.07) & $4.41(0.95)$ \\
\hline & & & & & \\
\hline & & NP & $4.36(0.82)$ & $4.14(1.01)$ & $4.20(0.94)$ \\
\hline \multirow{4}{*}{$\begin{array}{l}\text { Prime } \\
\text { Category }\end{array}$} & & $\mathrm{PP}$ & $4.61(0.74)$ & $4.41(1.02)$ & - \\
\hline & & NP & $4.27(1.05)$ & $4.01(1.01)$ & - \\
\hline & & $\mathrm{PP}$ & $4.54(0.79)$ & - & $4.41(1.03)$ \\
\hline & & NP & $4.17(0.88)$ & - & $4.14(0.92)$ \\
\hline
\end{tabular}

Notes. $\mathrm{PP}=$ positive prime $; \mathrm{NP}=$ negative prime. 
Table S9.

Experiment 4: Mean Rating Scores by Target Category, Prime Category, and Prime Valence (standard deviation in parentheses).

\begin{tabular}{|c|c|c|c|c|c|}
\hline & & & \multicolumn{3}{|c|}{ Target Category } \\
\hline & & & Dogs & Flowers & Food \\
\hline & & PP & $5.13(0.95)$ & $4.82(0.88)$ & $4.85(0.87)$ \\
\hline & & NP & $3.89(1.14)$ & $4.42(0.95)$ & $4.60(1.00)$ \\
\hline \multirow{5}{*}{$\begin{array}{l}\text { Prime } \\
\text { Category }\end{array}$} & & $\mathrm{PP}$ & $4.73(0.85)$ & $4.99(0.96)$ & $4.84(0.86)$ \\
\hline & & NP & $4.63(0.83)$ & $4.23(0.98)$ & $4.60(0.94)$ \\
\hline & & $\mathrm{PP}$ & $4.70(0.84)$ & $4.70(0.88)$ & $5.09(0.93)$ \\
\hline & Food & & & & \\
\hline & & NP & $4.63(0.83)$ & $4.45(0.92)$ & $4.53(0.98)$ \\
\hline
\end{tabular}

Notes. $\mathrm{PP}=$ positive prime; $\mathrm{NP}=$ negative prime.

\section{Additional significant effects in Experiments 2 and 4}

\section{Experiment 2:}

- A target category by prime valence interaction, $F(1,800)=74.16, p<.001, \eta_{\mathrm{p}}^{2}=.08$, indicated a stronger prime valence effect when the targets were landscapes, $F(1,400)=$ $399.10, p<.001, \eta_{\mathrm{p}}{ }^{2}=.50$, than when the targets were men, $F(1,400)=144.22, p<.001, \eta_{\mathrm{p}}{ }^{2}$ $=.27$.

- A prime category by target category interaction, $F(1,800)=23.85, p<.001, \eta_{\mathrm{p}}^{2}=.03$, indicated that when the targets were landscapes, participants rated targets that appeared after 
landscape primes considerably more positively than targets that appeared after people primes , $F(1,400)=95.67, p<.001, \eta_{\mathrm{p}}^{2}=.19$, whereas when the targets were people, this effect was attenuated, $F(1,400)=32.01, p<.001, \eta_{\mathrm{p}}^{2}=.07$.

- A weak but significant target category by target medium interaction, $F(1,800)=4.45, p=$ $.035, \eta_{\mathrm{p}}^{2}=.01$, indicated that when the targets were landscapes, participants rated sketch targets with similar positivity to photographic targets, $F(1,400)=1.66, p=.199, \eta_{\mathrm{p}}^{2}<.01$, whereas when the targets were people, participants rated sketch targets more positively than photographic targets, $F(1,400)=22.81, p<.001, \eta_{\mathrm{p}}{ }^{2}=.05$.

- The target category by target medium interaction was qualified by a three-way interaction with prime valence, $F(1,800)=58.07, p<.001, \eta_{p}{ }^{2}=.07$, indicating that when the prime was positive, the aforementioned pattern of interaction between target category and target medium appeared, $F(1,800)=44.76, p<.001, \eta_{\mathrm{p}}{ }^{2}=.05$. When the prime was negative, on the other hand, the interaction between target category and target medium, $F(1,800)=7.87$, $p=.005, \eta_{\mathrm{p}}^{2}=.01$, indicated that, when the targets were landscapes, participants rated sketches more positively than photographic targets, $F(1,400)=22.42, p<.001, \eta_{\mathrm{p}}{ }^{2}=.05$, whereas when the targets were people, participants rated sketches and photographic targets similarly, $F(1,800)=2.17, p=.142, \eta_{\mathrm{p}}^{2}=.01$.

- A prime category by prime valence interaction, $F(1,800)=12.58, p<.001, \eta_{\mathrm{p}}{ }^{2}=.02$, reflected a stronger prime valence effect when the primes were landscapes, $F(1,800)=$ $457.76, p<.001, \eta_{\mathrm{p}}{ }^{2}=.36$, than when the primes were men, $F(1,800)=236.03, p<.001, \eta_{\mathrm{p}}{ }^{2}$ $=.23$.

- The prime valence by prime category interaction was qualified by a three-way interaction with target medium, $F(1,800)=42.10, p<.001, \eta_{p}^{2}=.05$, indicating that when the targets 
were photographs there was a larger effect of prime valence when the primes were landscapes, $F(1,403)=42.70, p<.001, \eta_{p}{ }^{2}=.10$, but when the targets were sketches the effect of prime valence was somewhat larger when the primes were men, $F(1,397)=4.56, p$ $=.033, \eta_{p}^{2}=.01$.

\section{Experiment 4:}

- A prime category by prime valence interaction, $F(1,1672)=41.95, p<.001, \eta_{\mathrm{p}}^{2}=.05$, indicated a stronger prime valence effect when the primes were dogs, $F(1,836)=235.11, p<$ $.001, \eta_{\mathrm{p}}{ }^{2}=.22$, than when the primes were flowers, $F(1,836)=163.70, p<.001, \eta_{\mathrm{p}}^{2}=.16$, or food, $F(1,836)=111.79, p<.001, \eta_{\mathrm{p}}^{2}=.12$.

- A prime category by target category interaction, $F(1,1672)=11.21, p<.001, \eta_{\mathrm{p}}^{2}=.03$, indicated that, when the targets were food, participants rated the targets more positively after food primes than after flower and dog primes, $F(1,590)=6.30, p<.001, \eta_{\mathrm{p}}{ }^{2}=.02$, whereas when the targets were flowers, participants rated the targets numerically less positively after food primes than after the other prime types, $F(1,512)=1.32, p<.001, \eta_{\mathrm{p}}^{2}=.01$, and when the targets were dogs, participants rated targets that appeared after dog primes less positively than the targets that appeared after other primes types, $F(1,570)=26.83, p<.001, \eta_{\mathrm{p}}{ }^{2}=.09$. 
Testing the Pleasantness of Prime Stimuli

Table S10. Experiments 1-4: Mean pleasantness ratings for all prime sets.

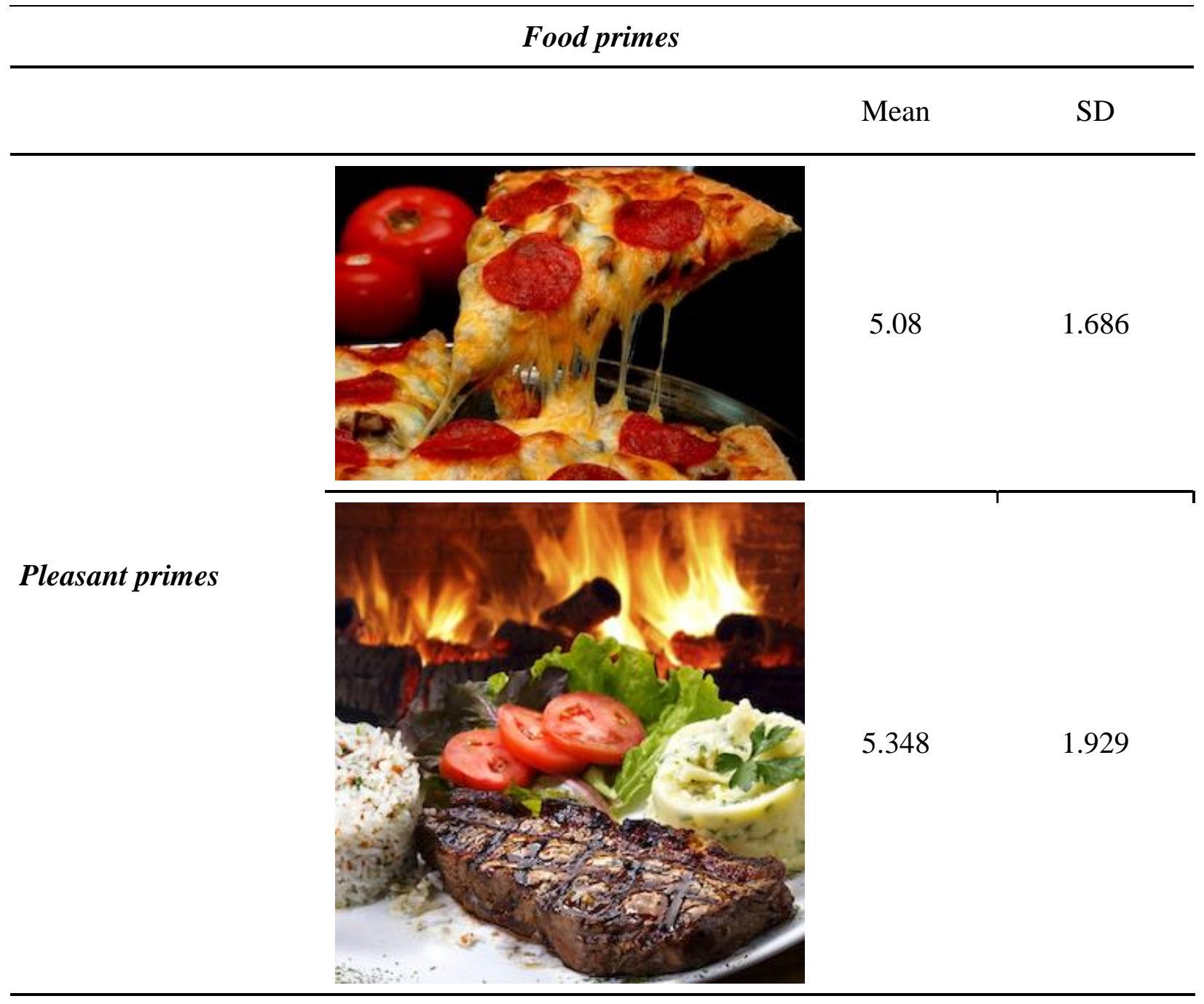




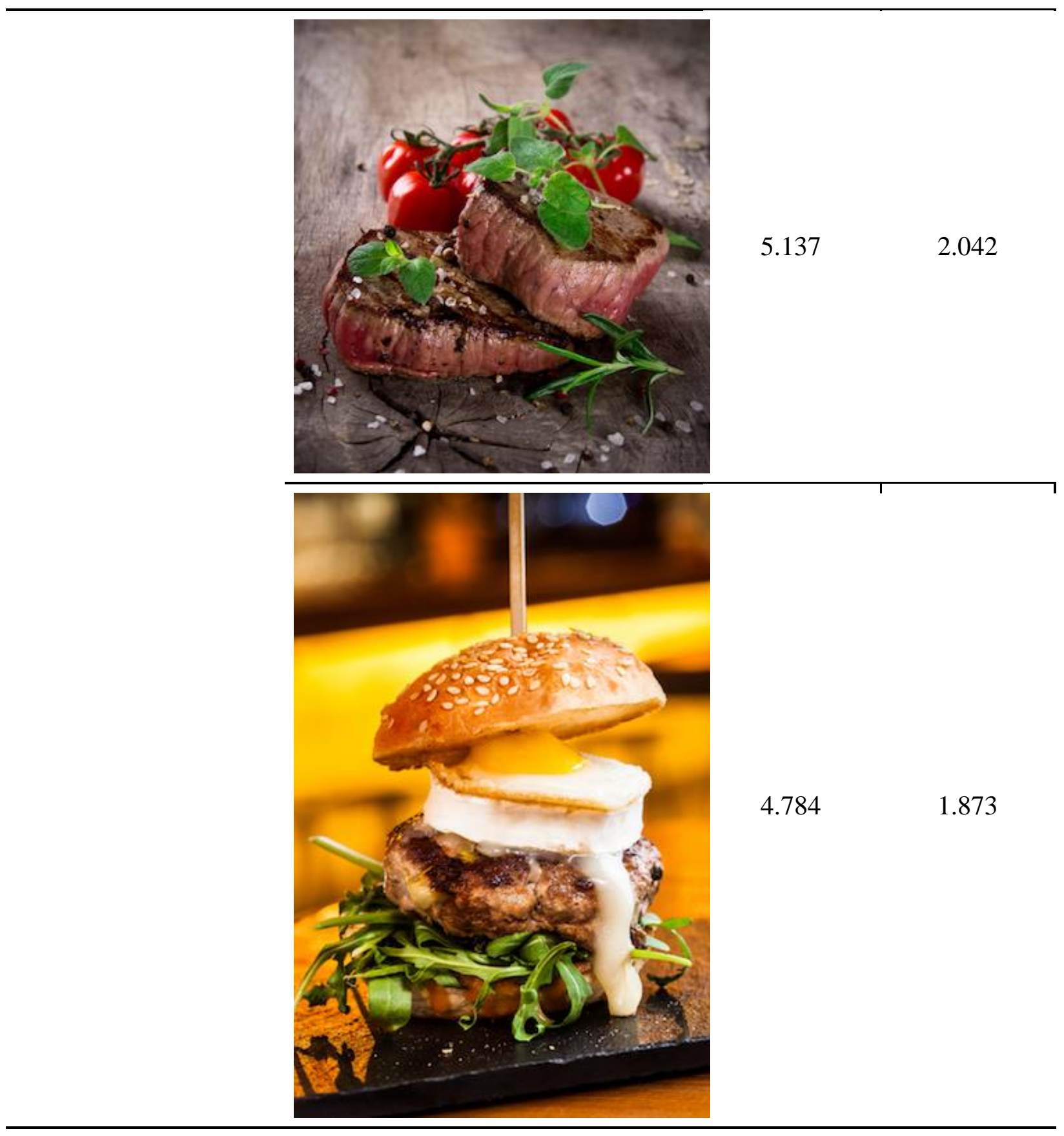




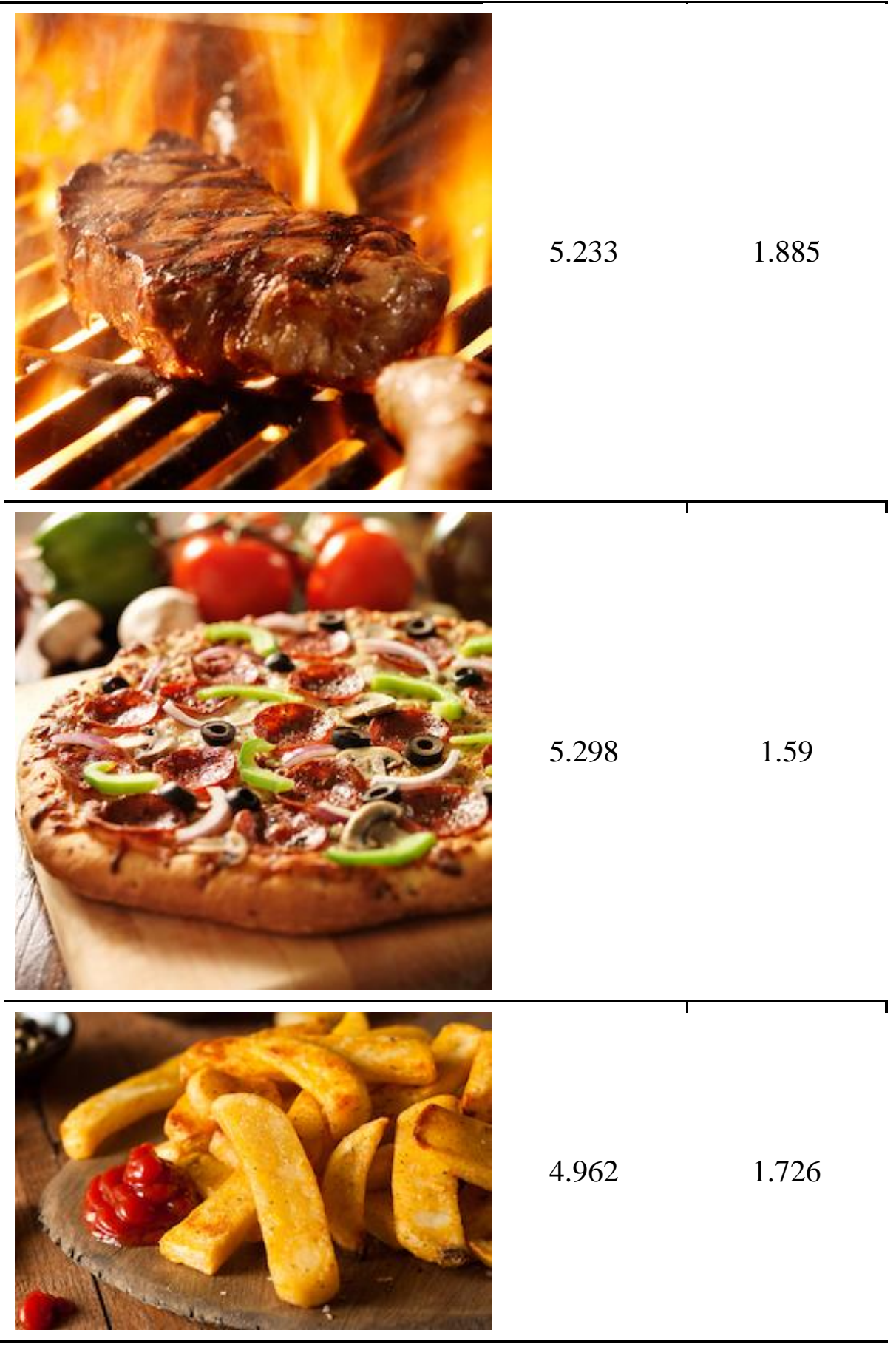




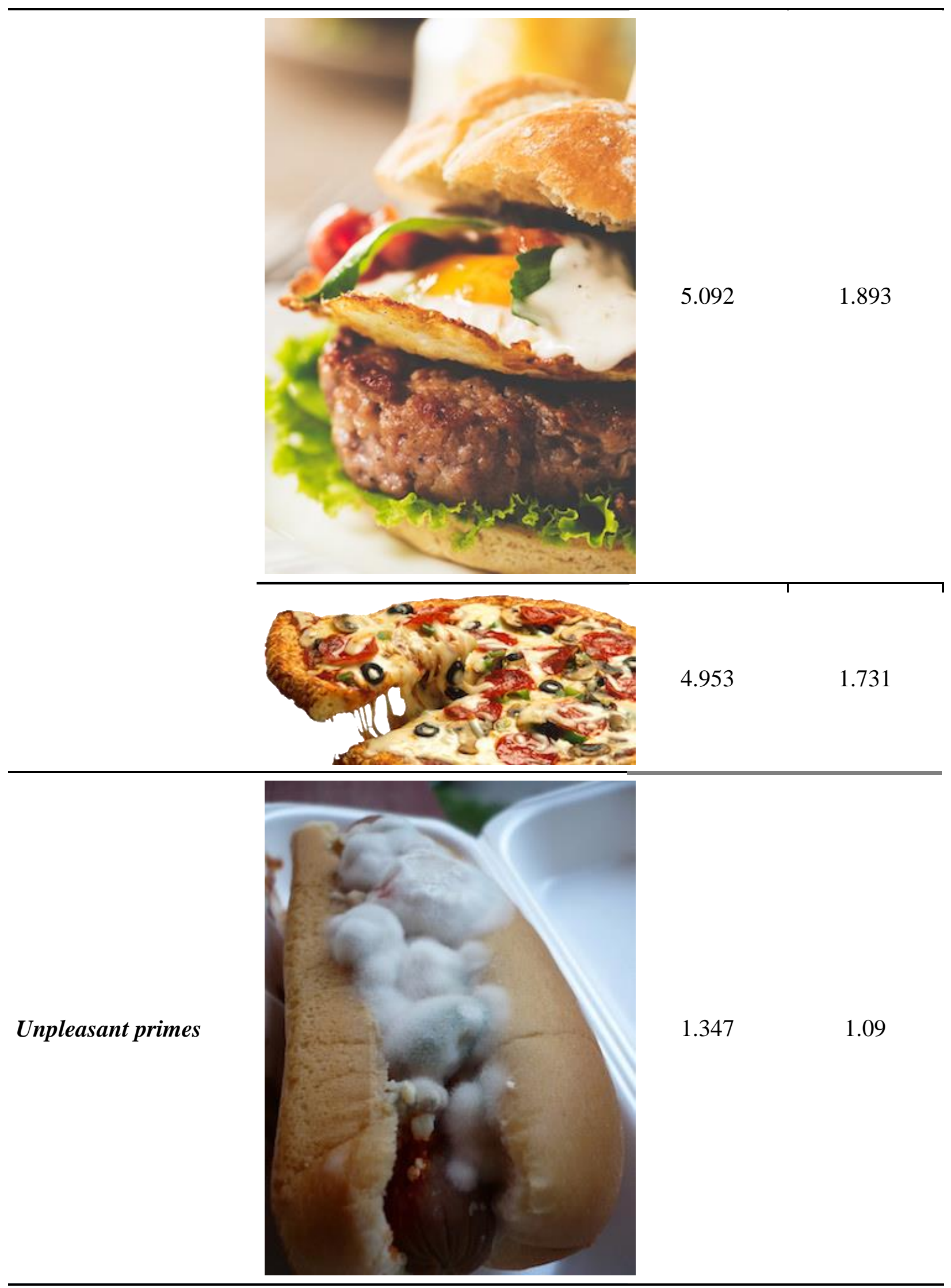



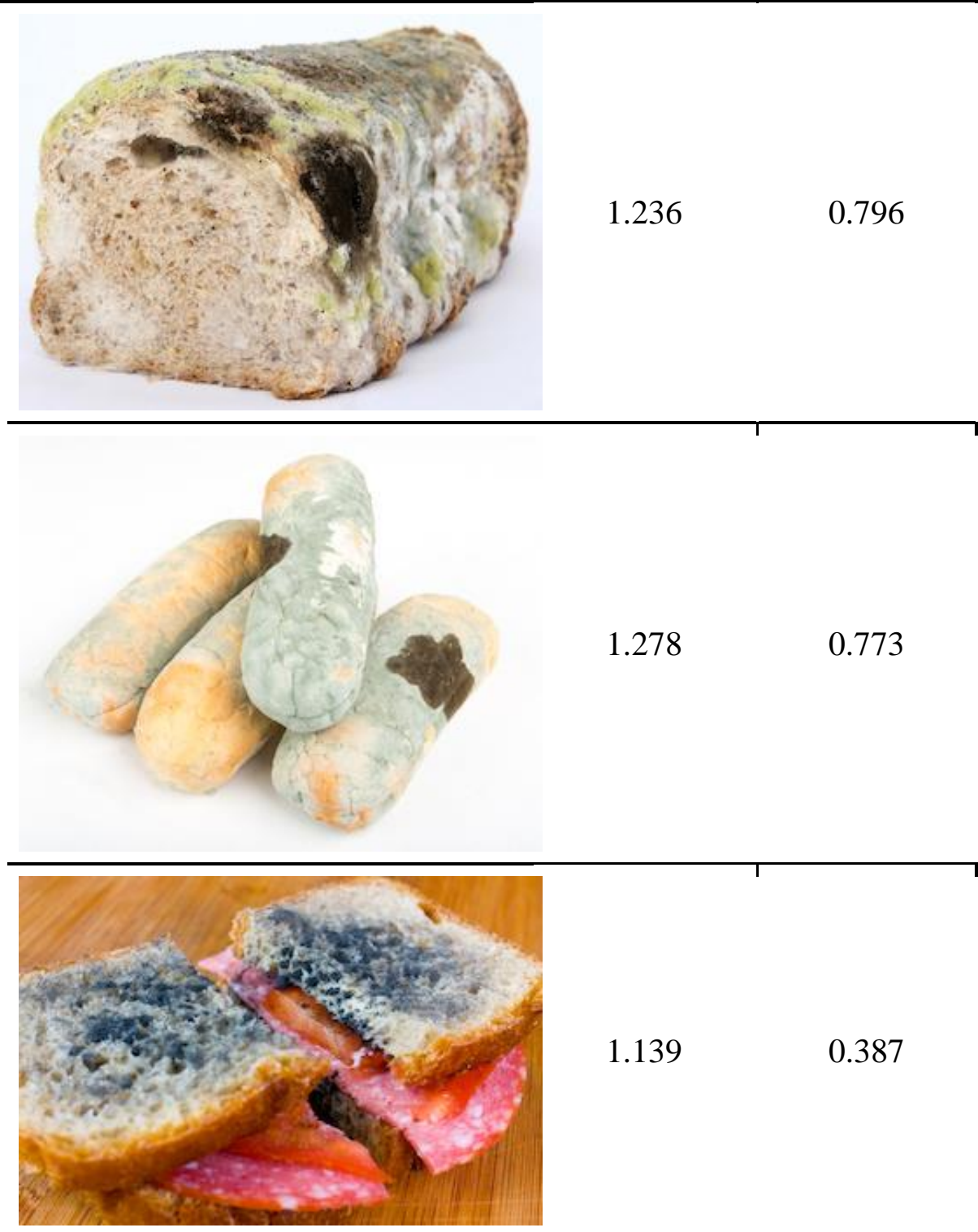

$\begin{array}{ll}1.139 & 0.387\end{array}$

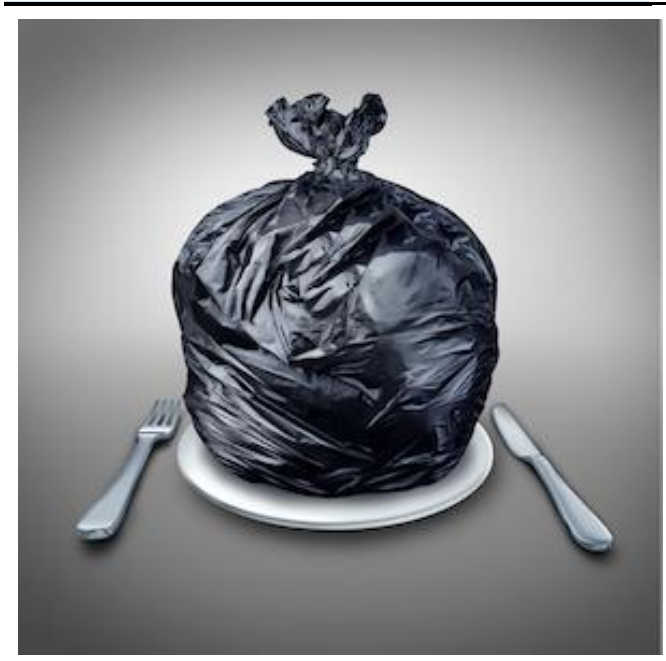

1.75

1.058 

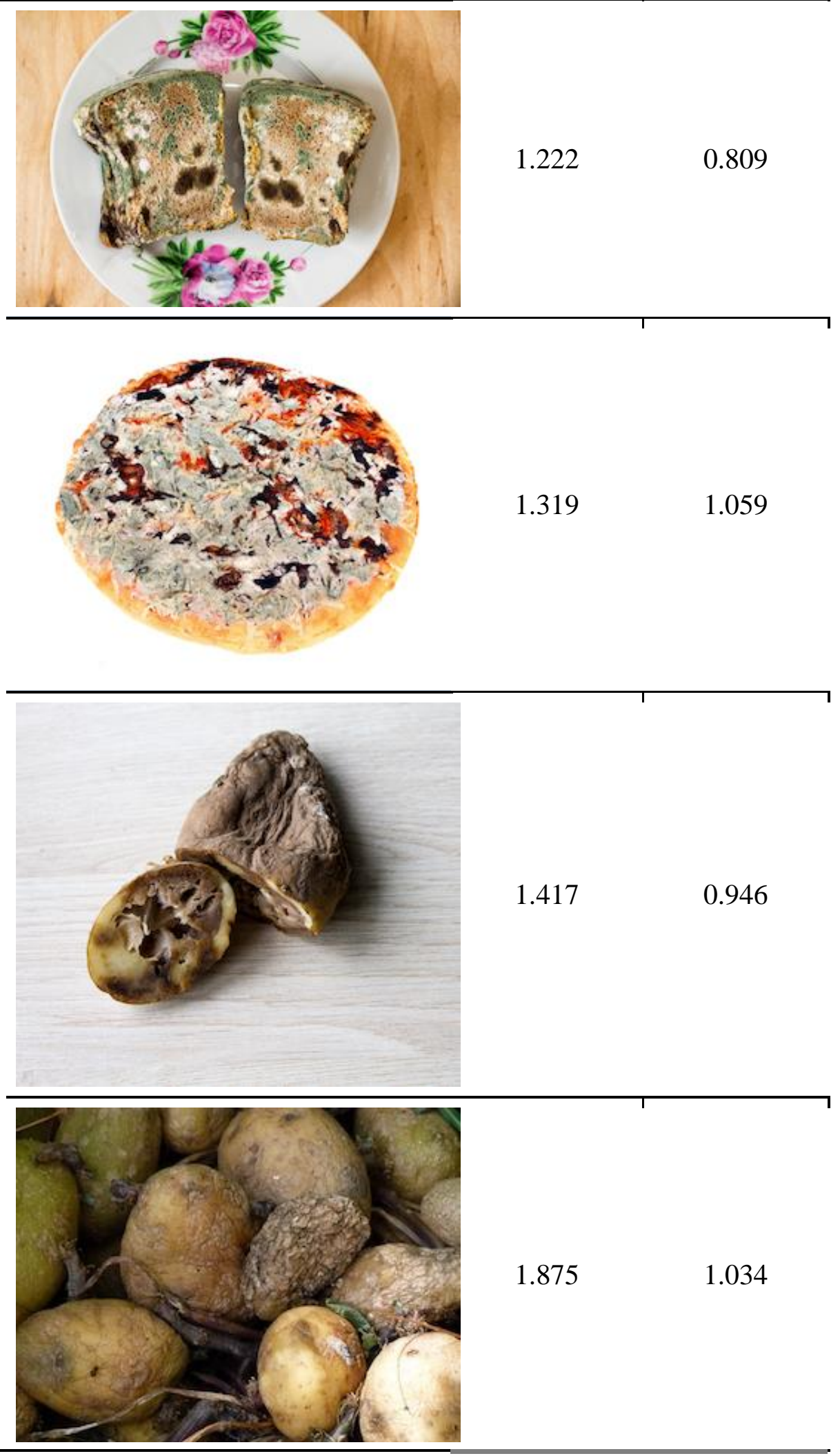

Dog primes 


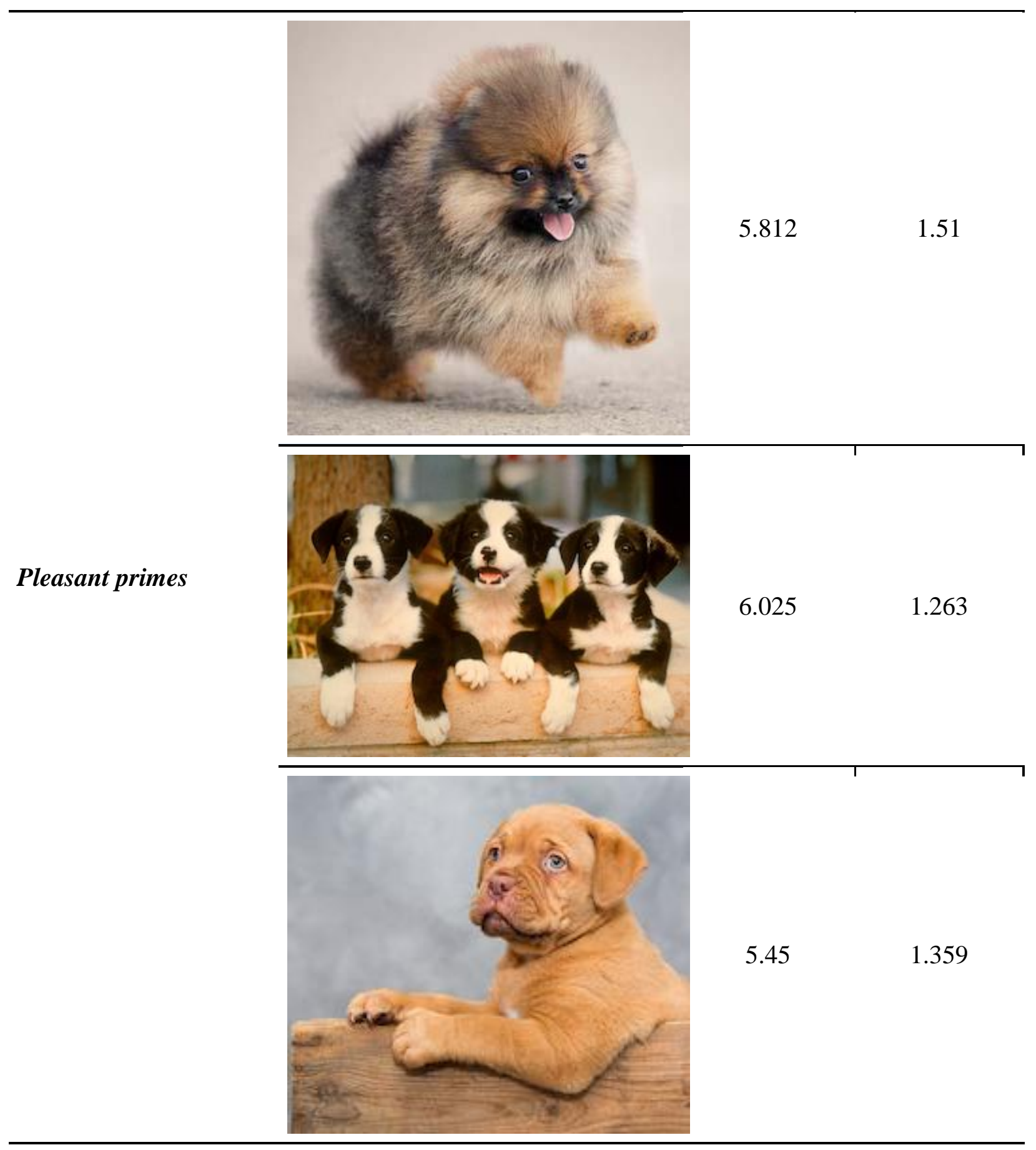




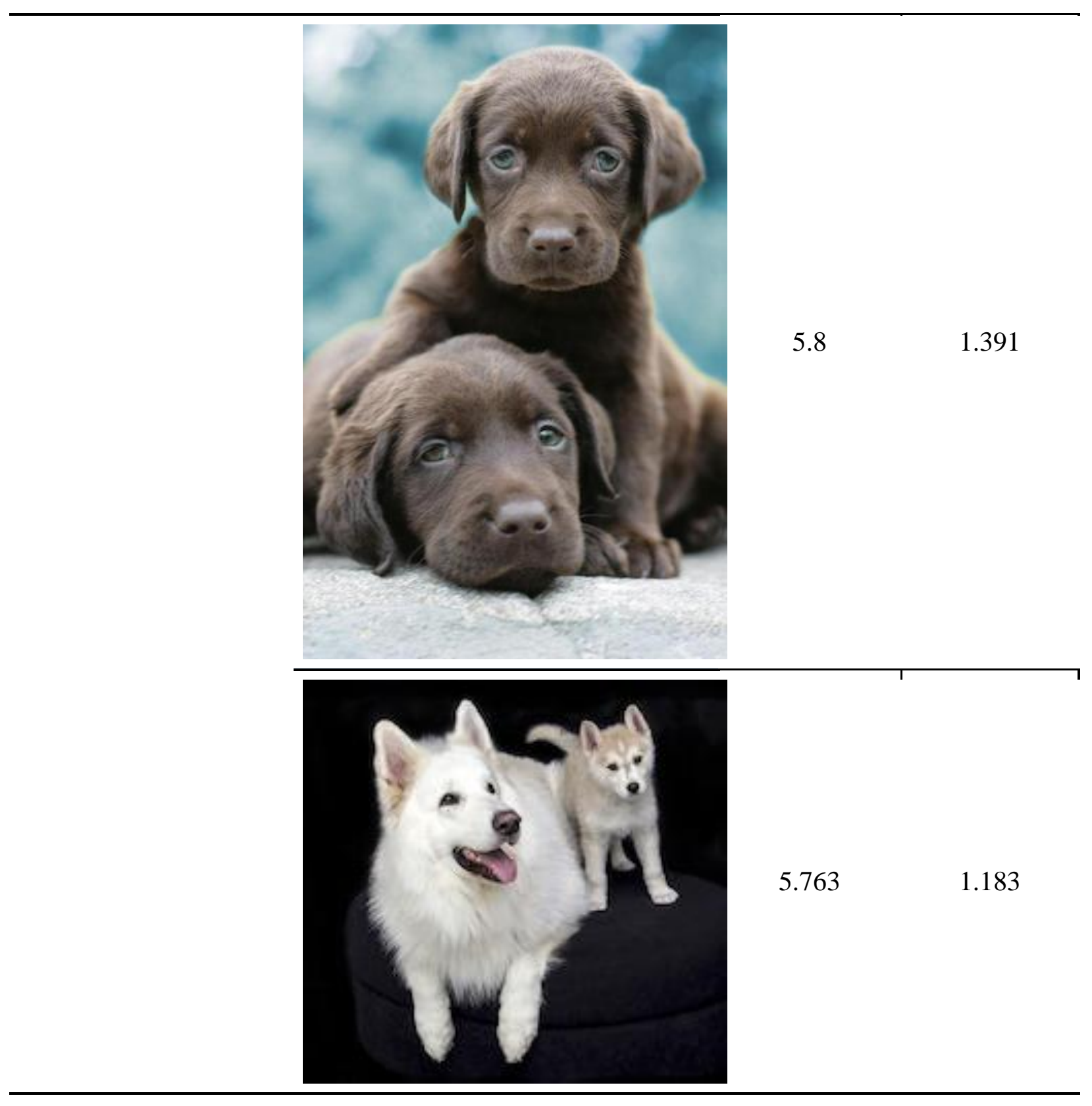




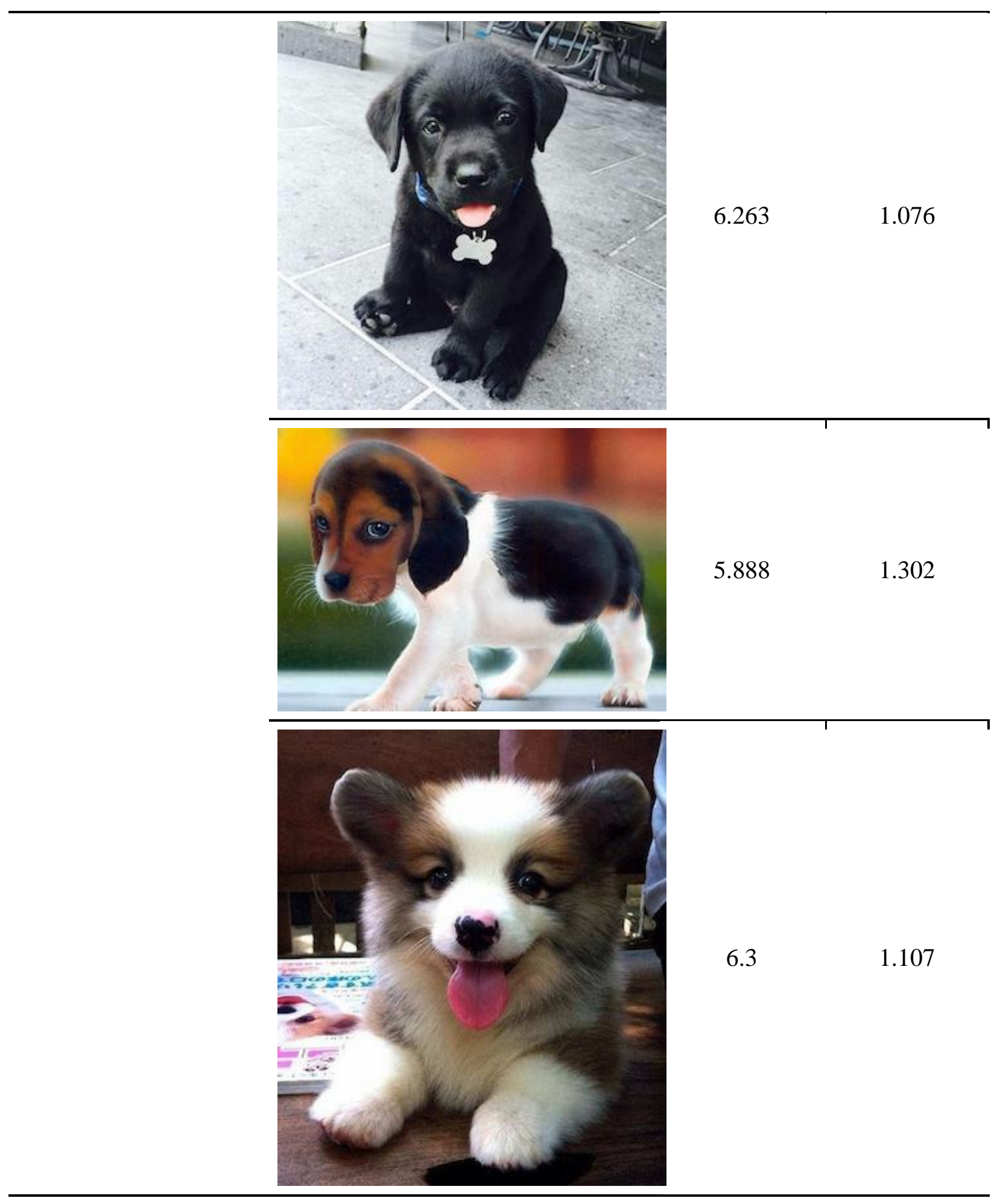




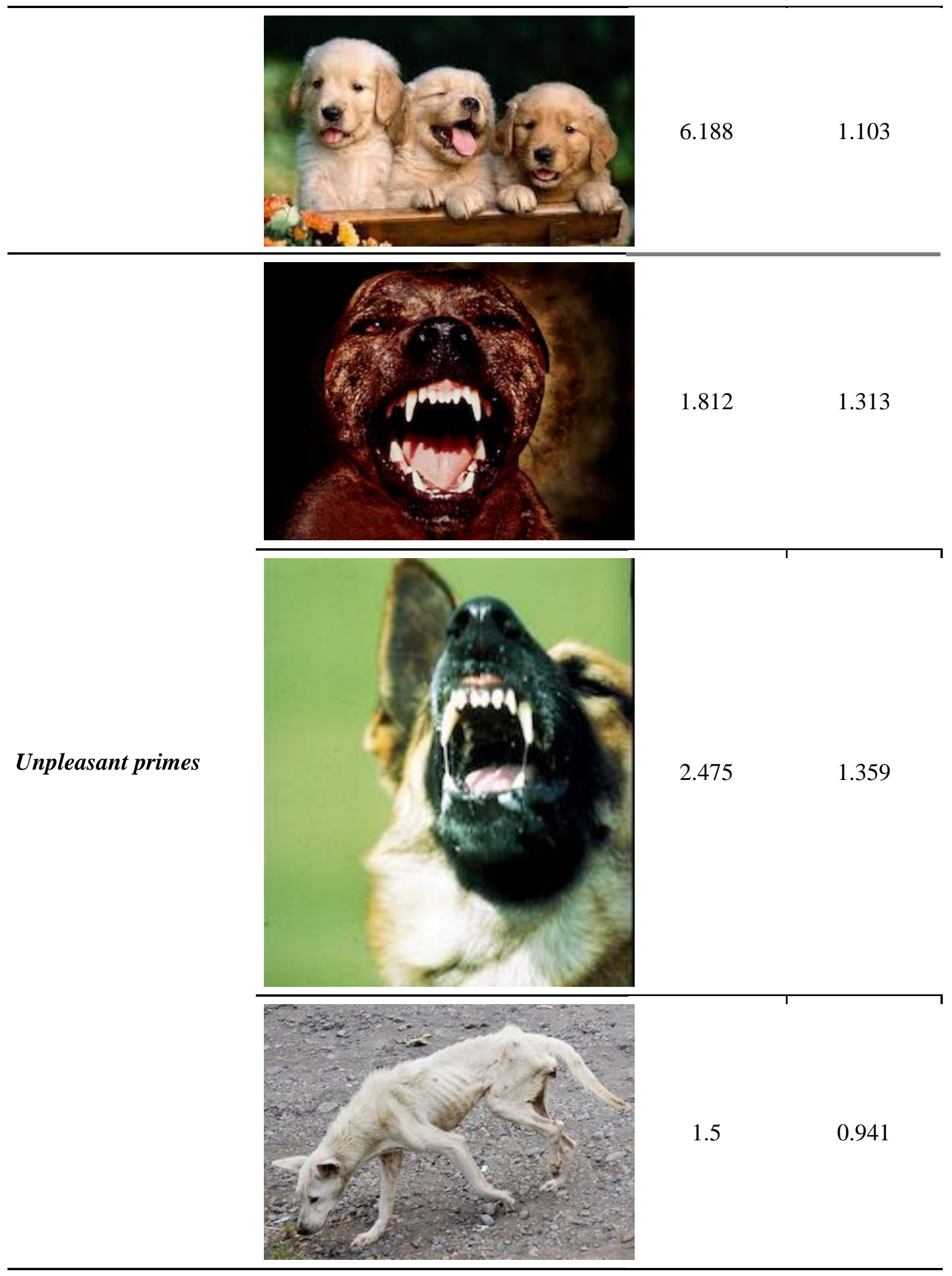




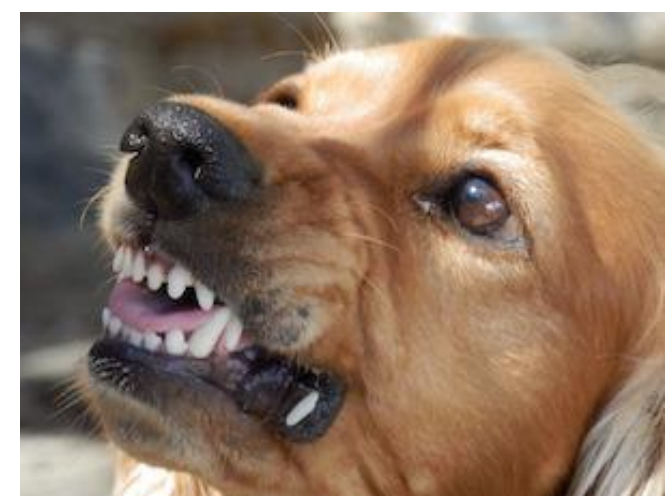

$3.487 \quad 1.526$

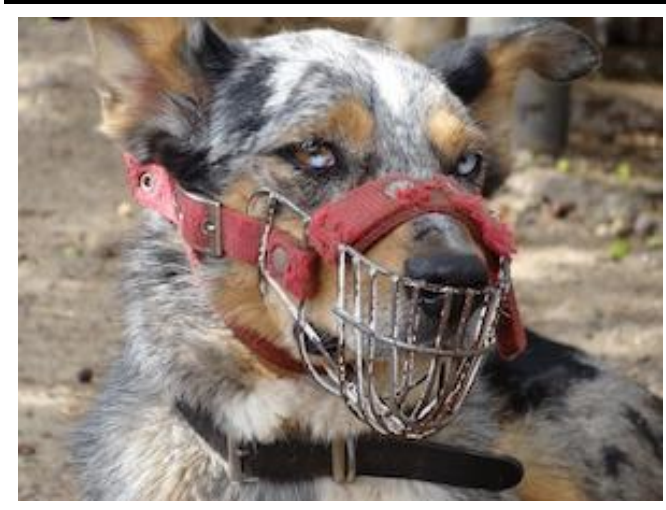

1.788

1.166
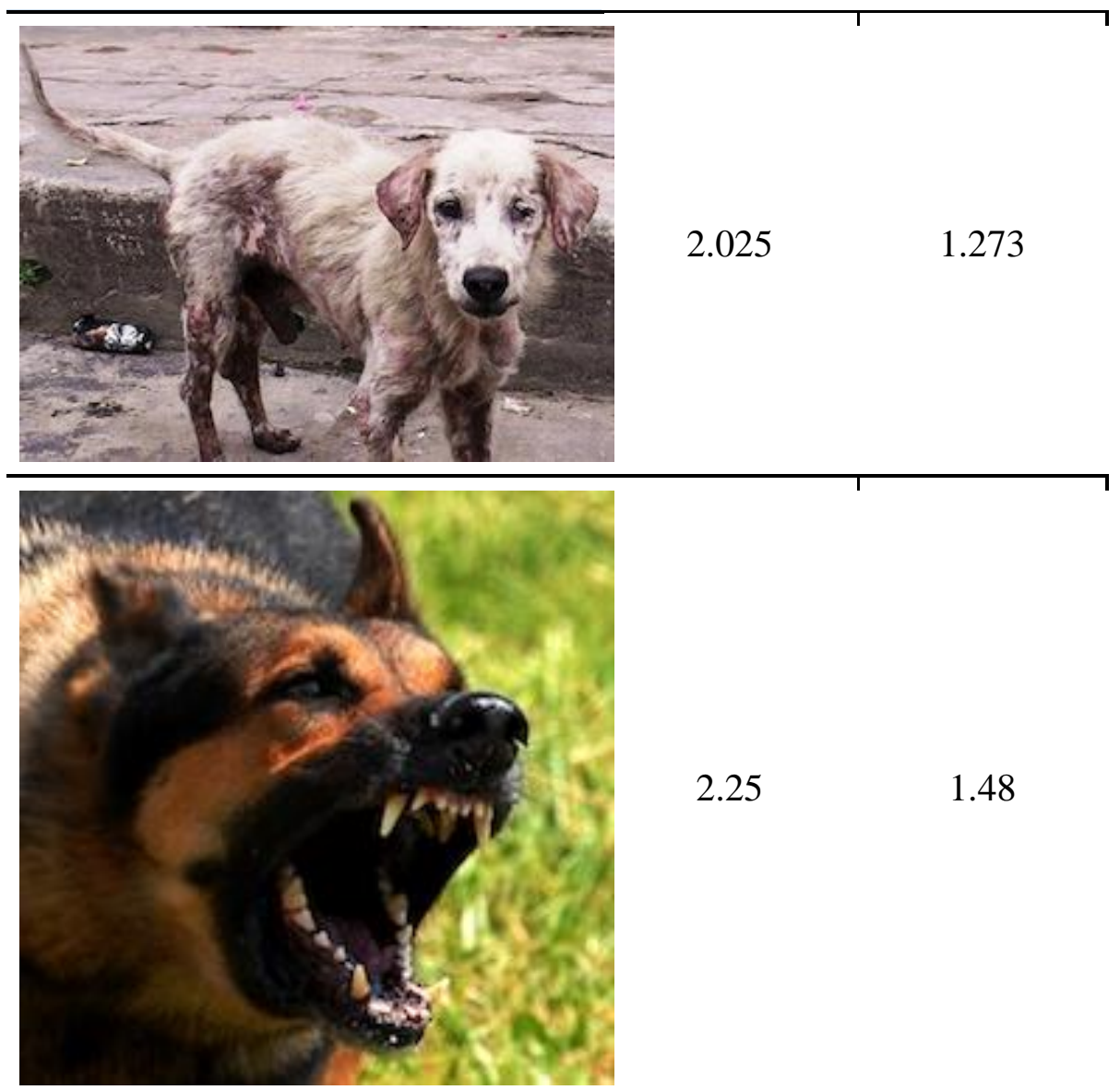

2.25

1.48 


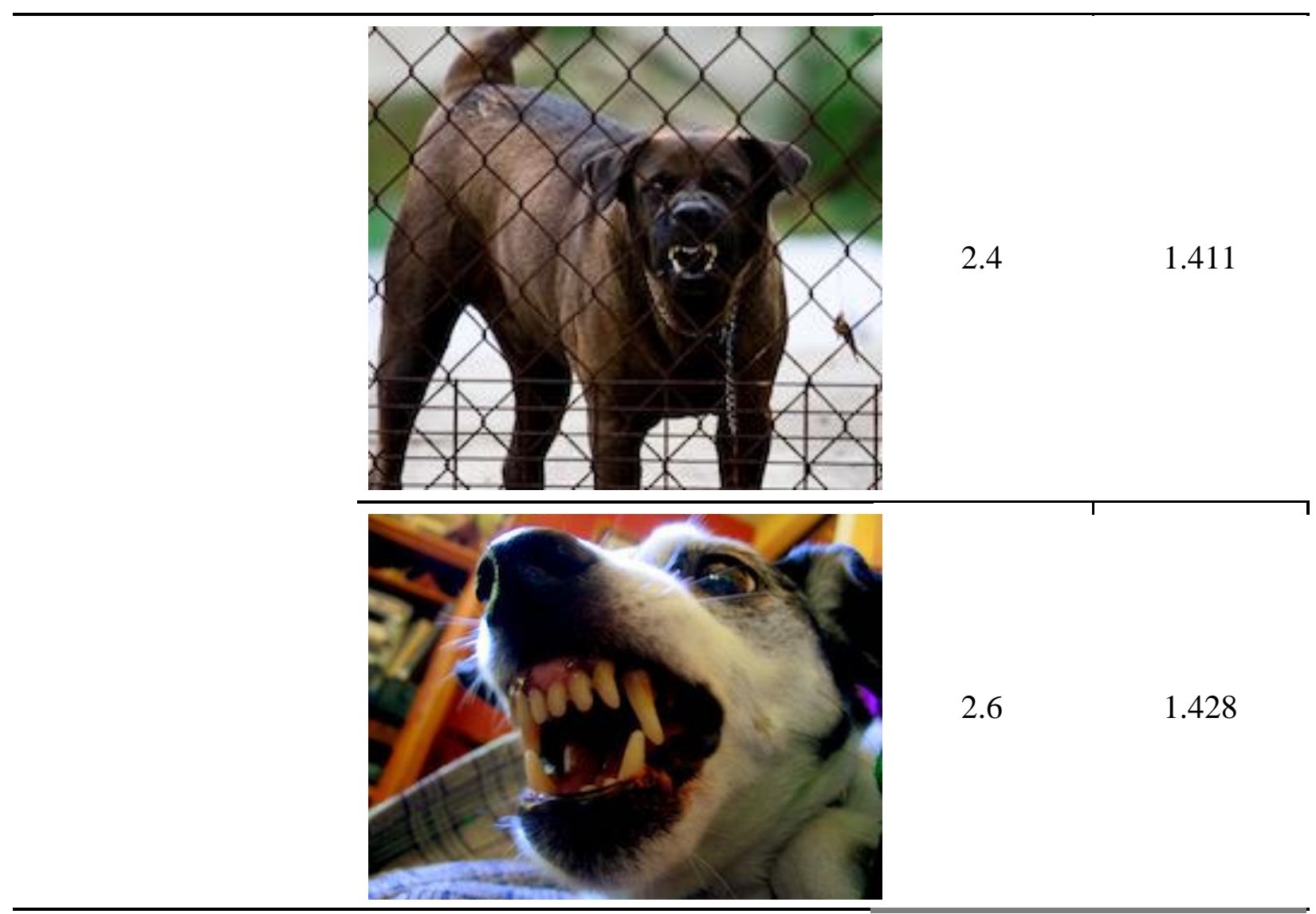

Flower primes

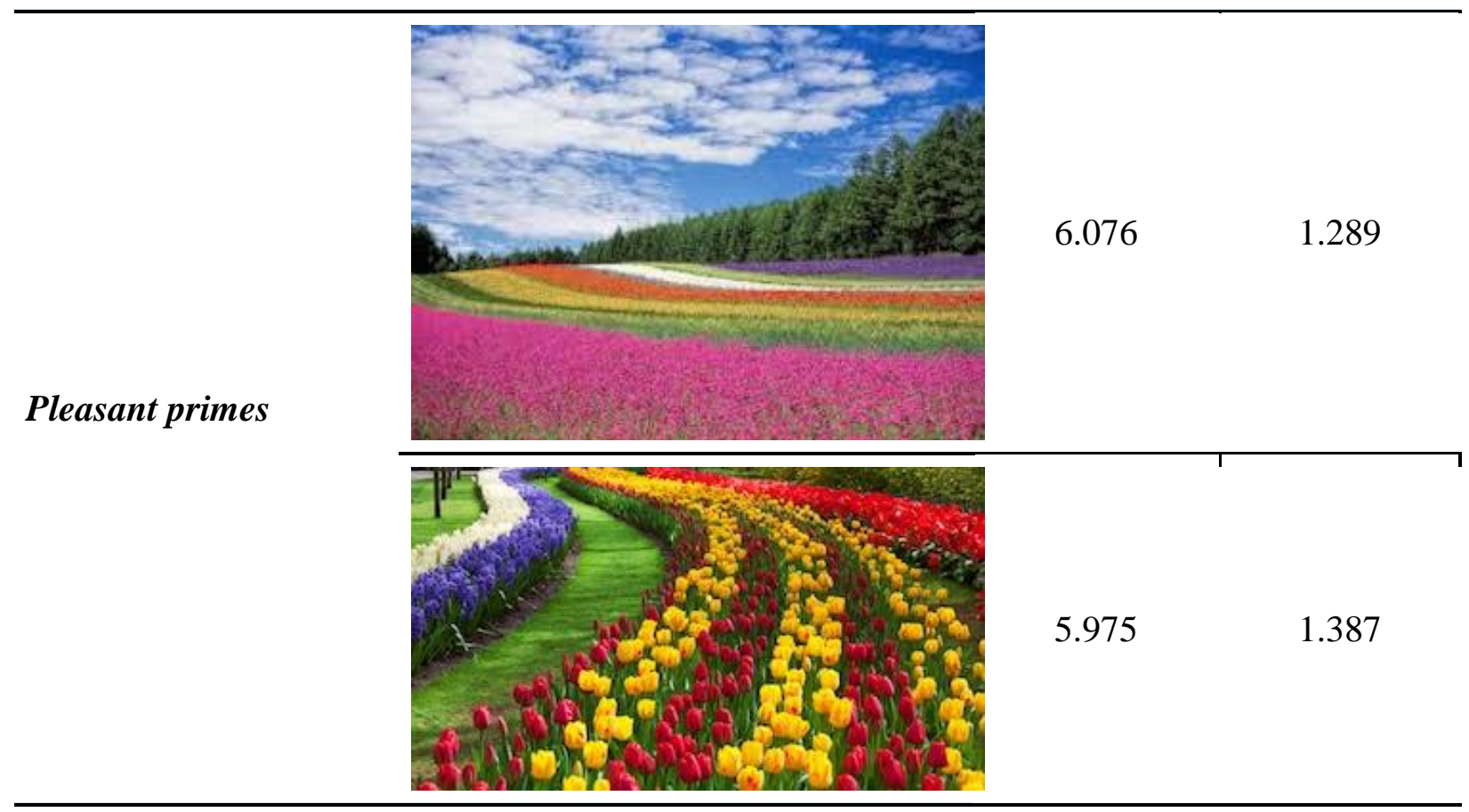




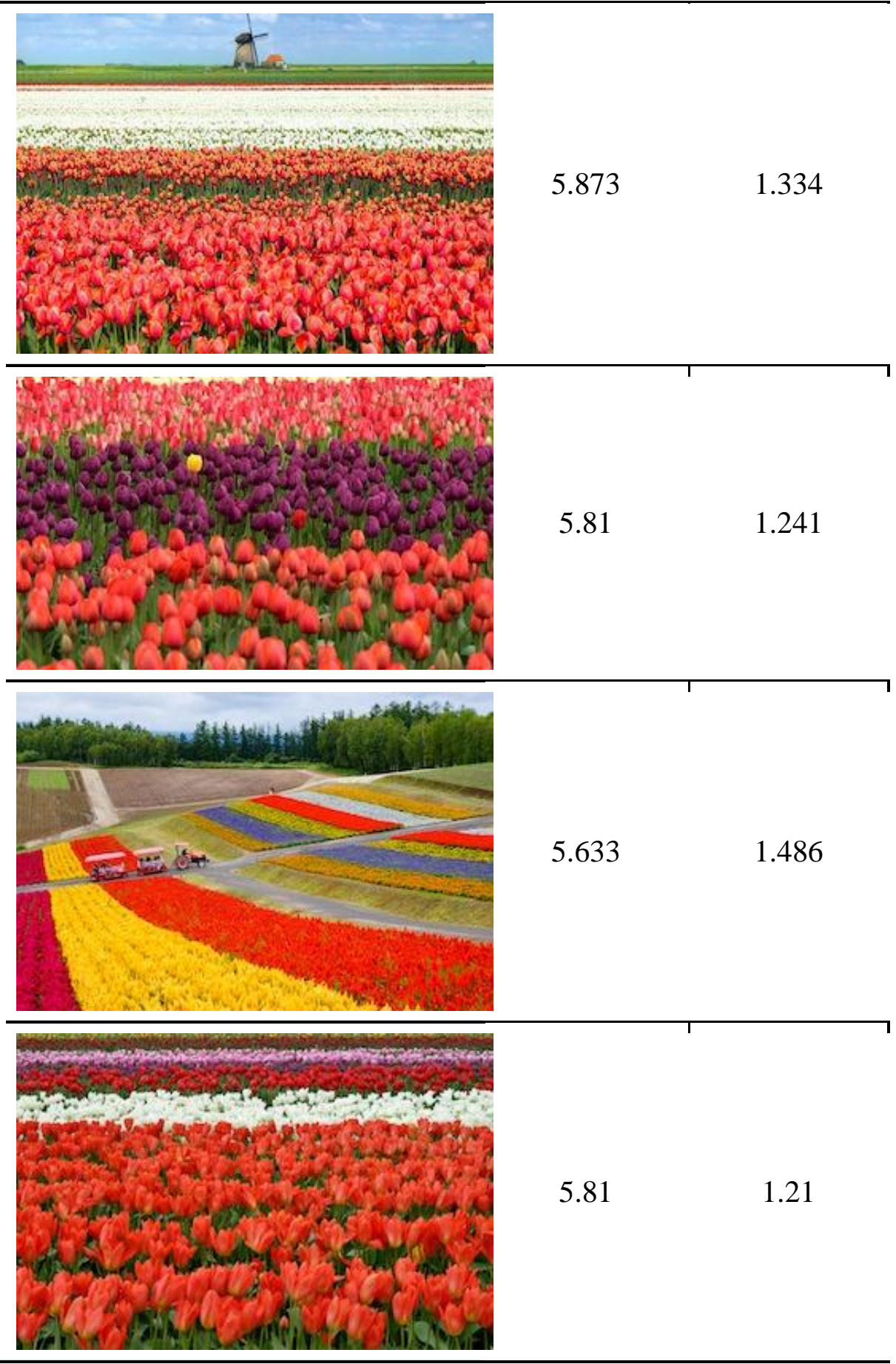




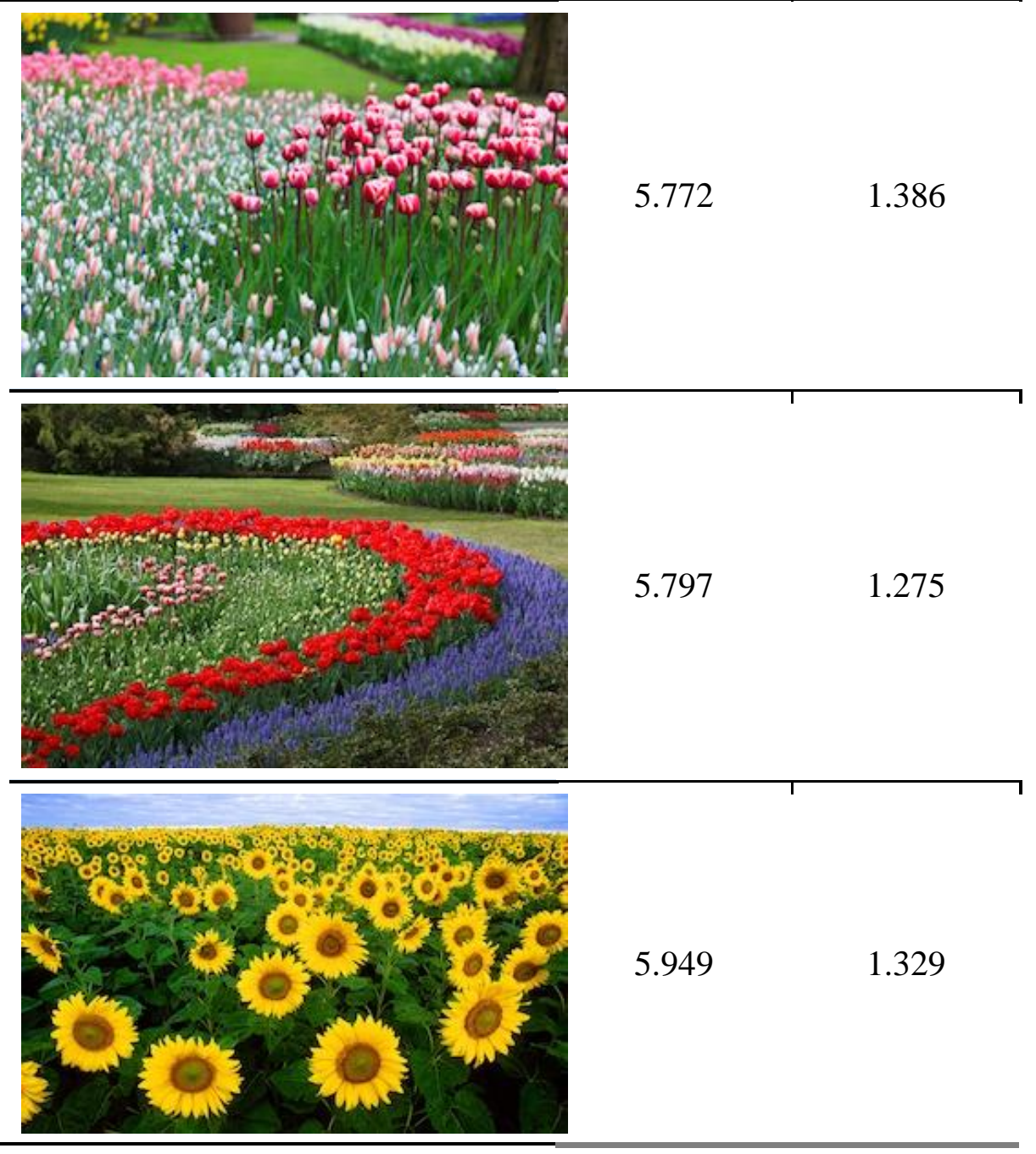

Unpleasant primes

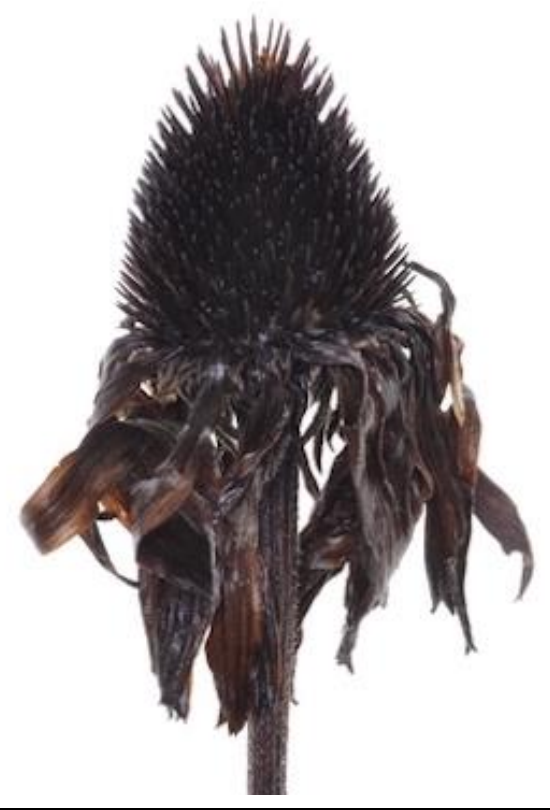

2.443

1.43 


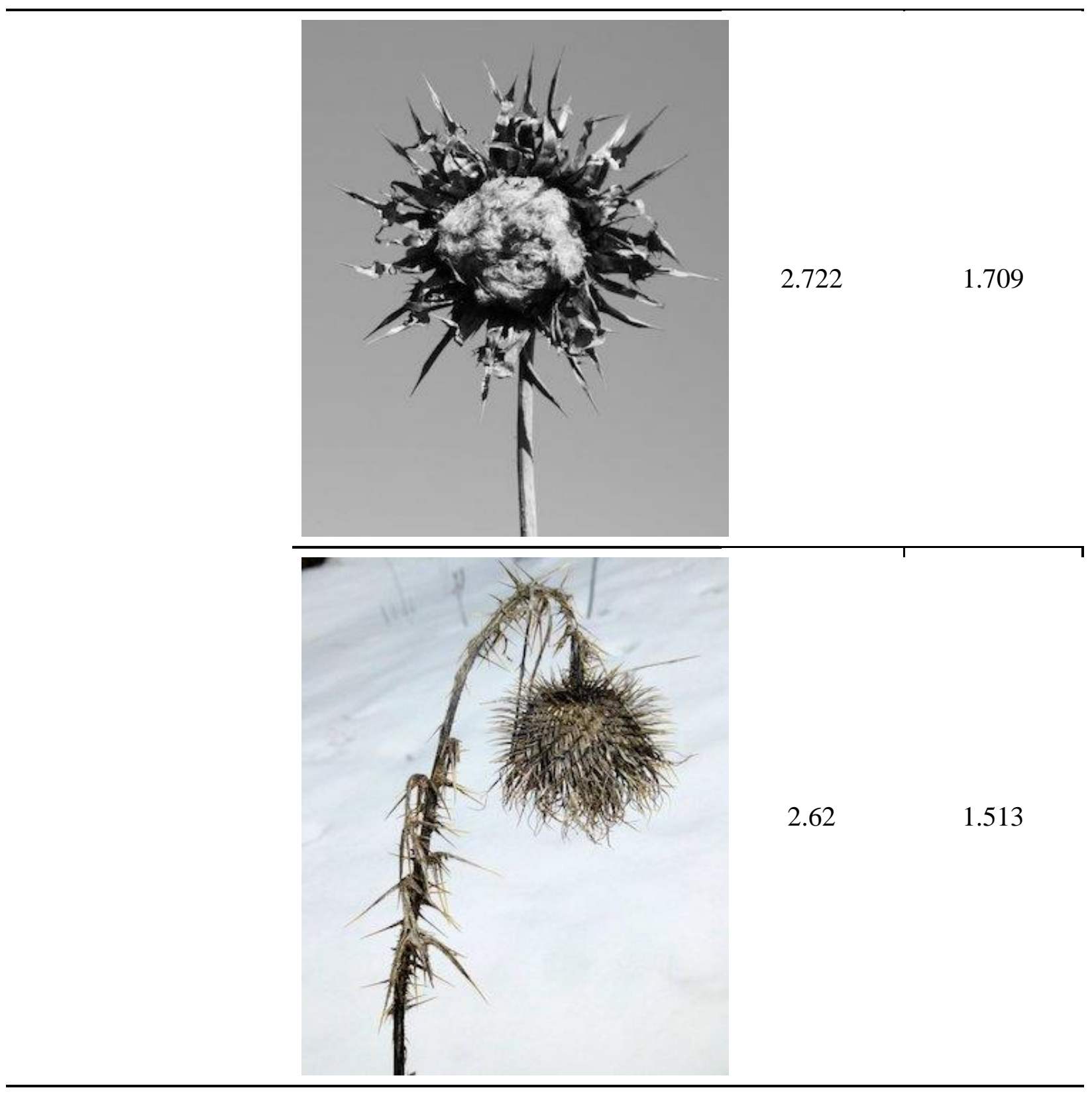




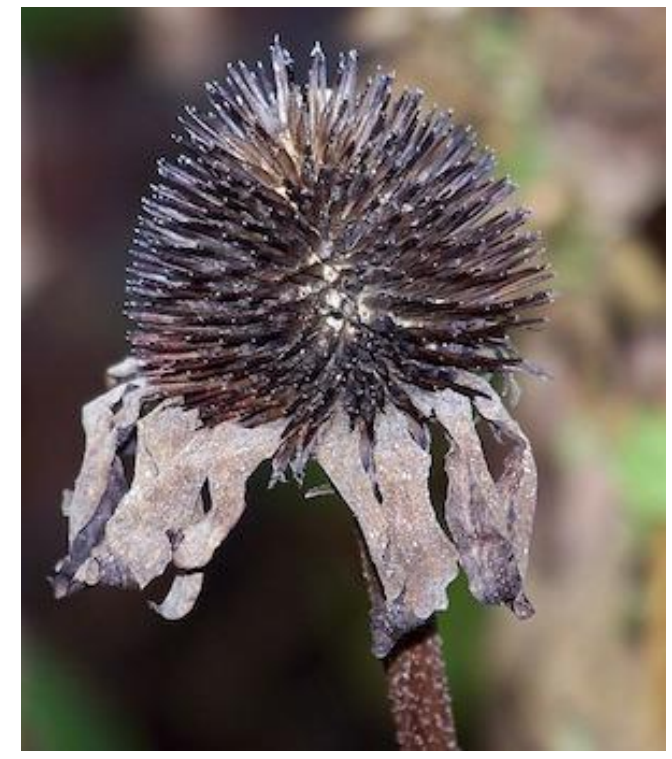
2.911
1.619

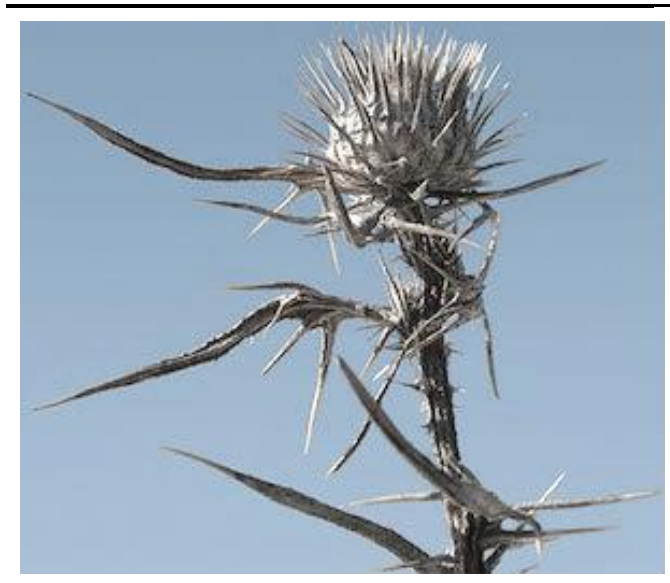

2.81

1.641

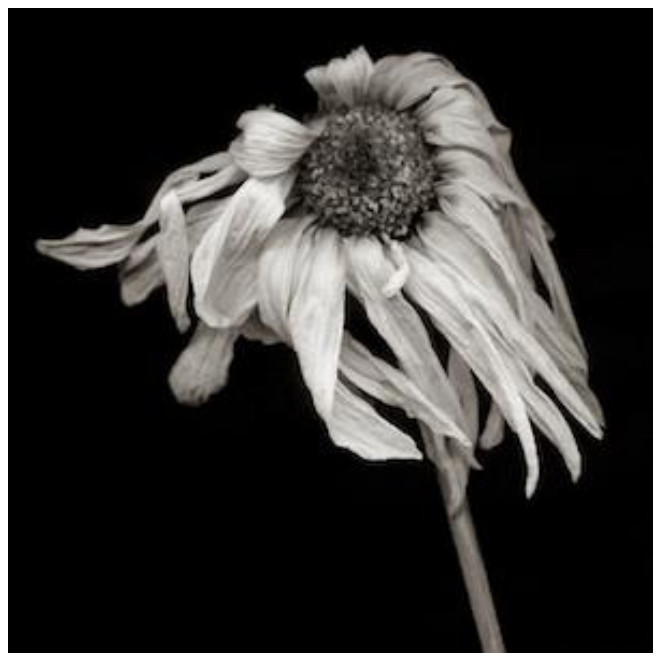

3.165

1.548 


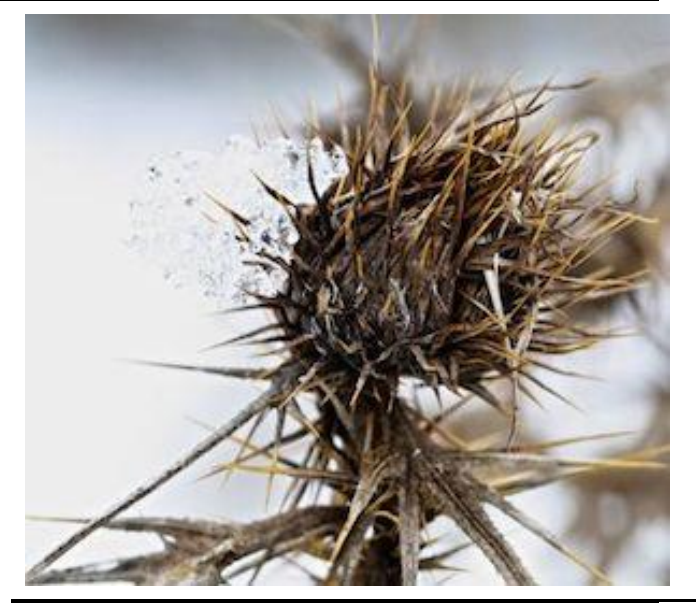

2.709

1.52

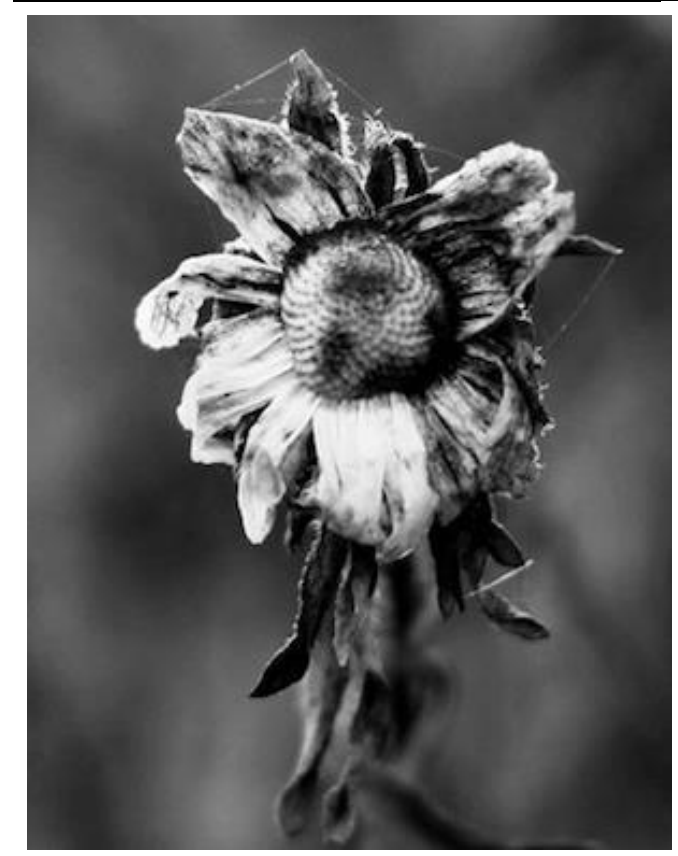

2.62

1.47

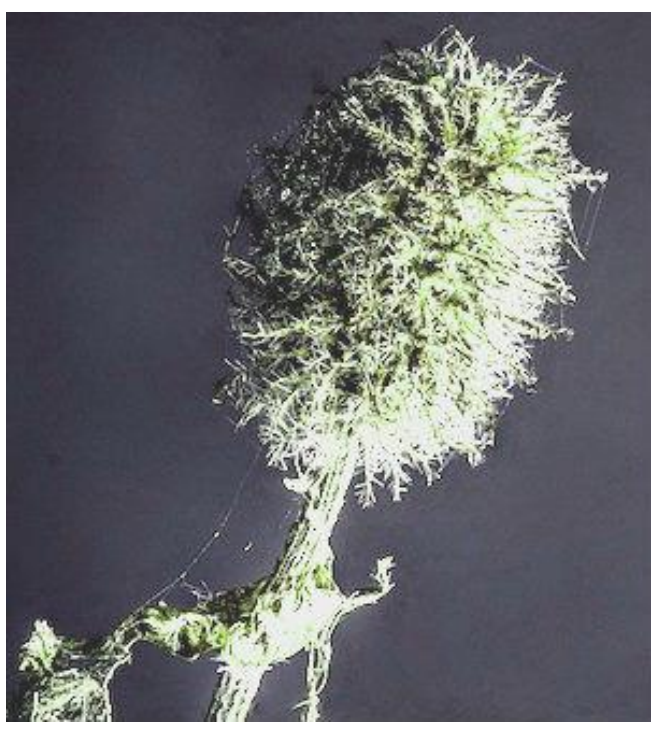

2.886

1.672 


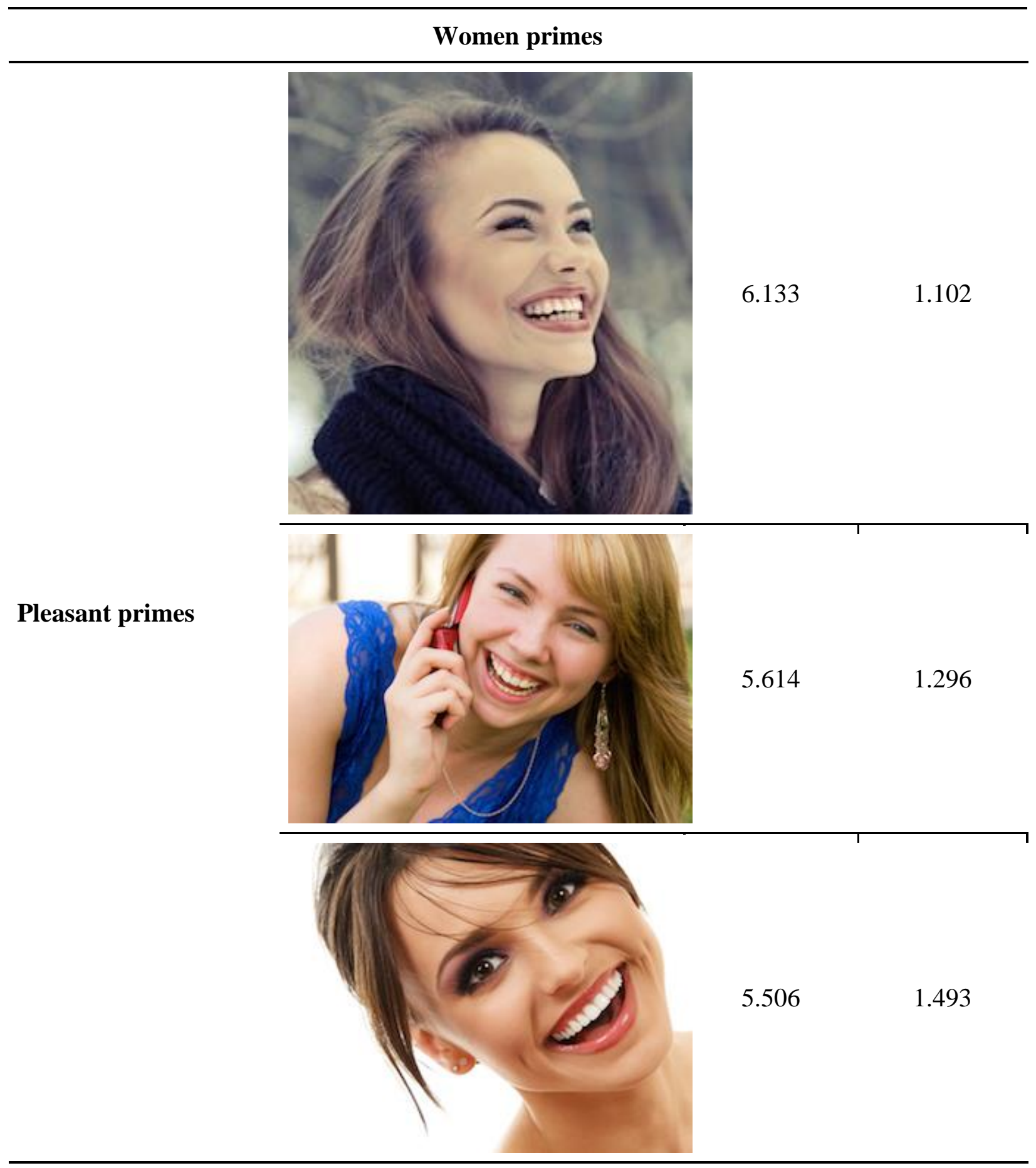




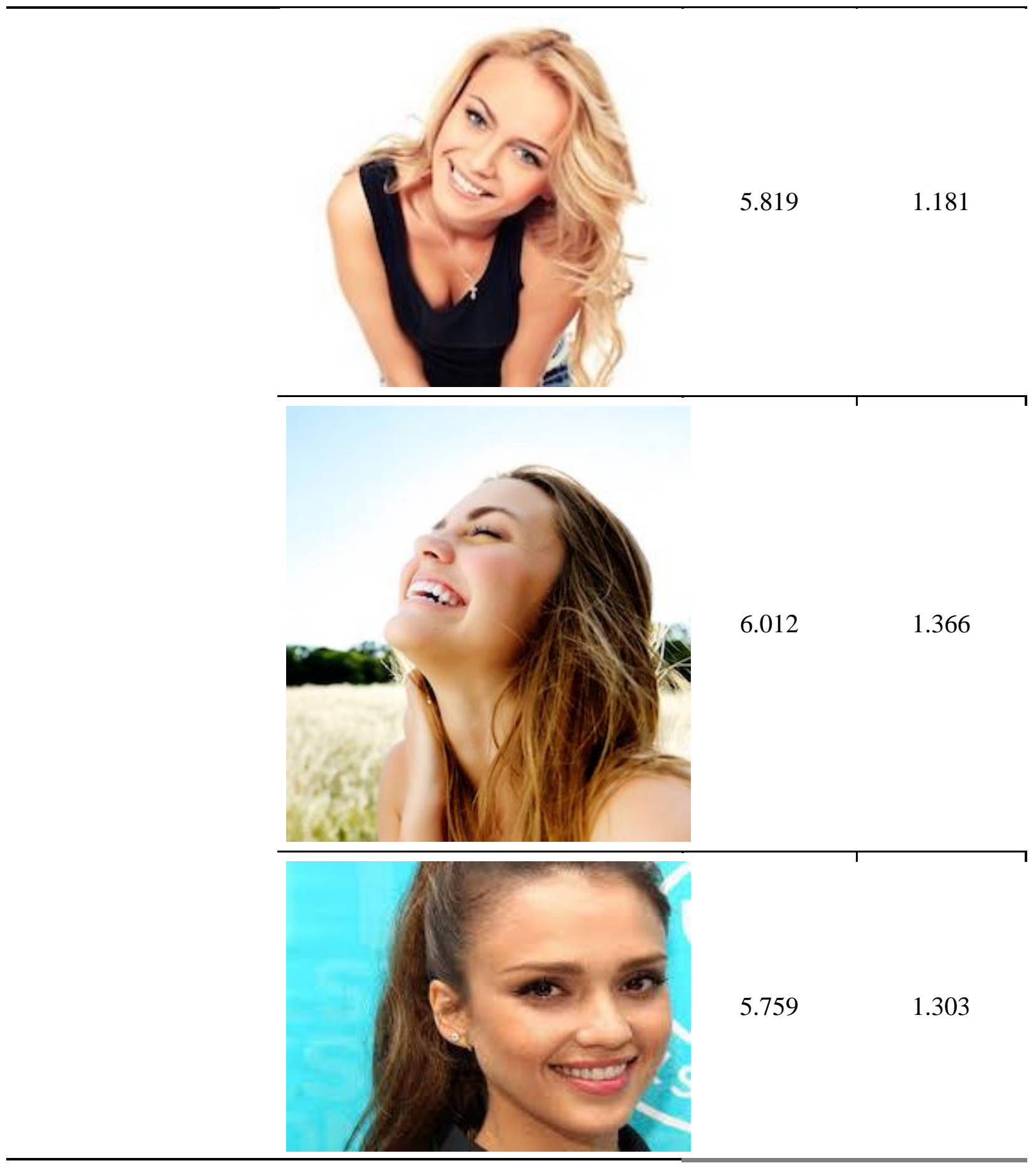




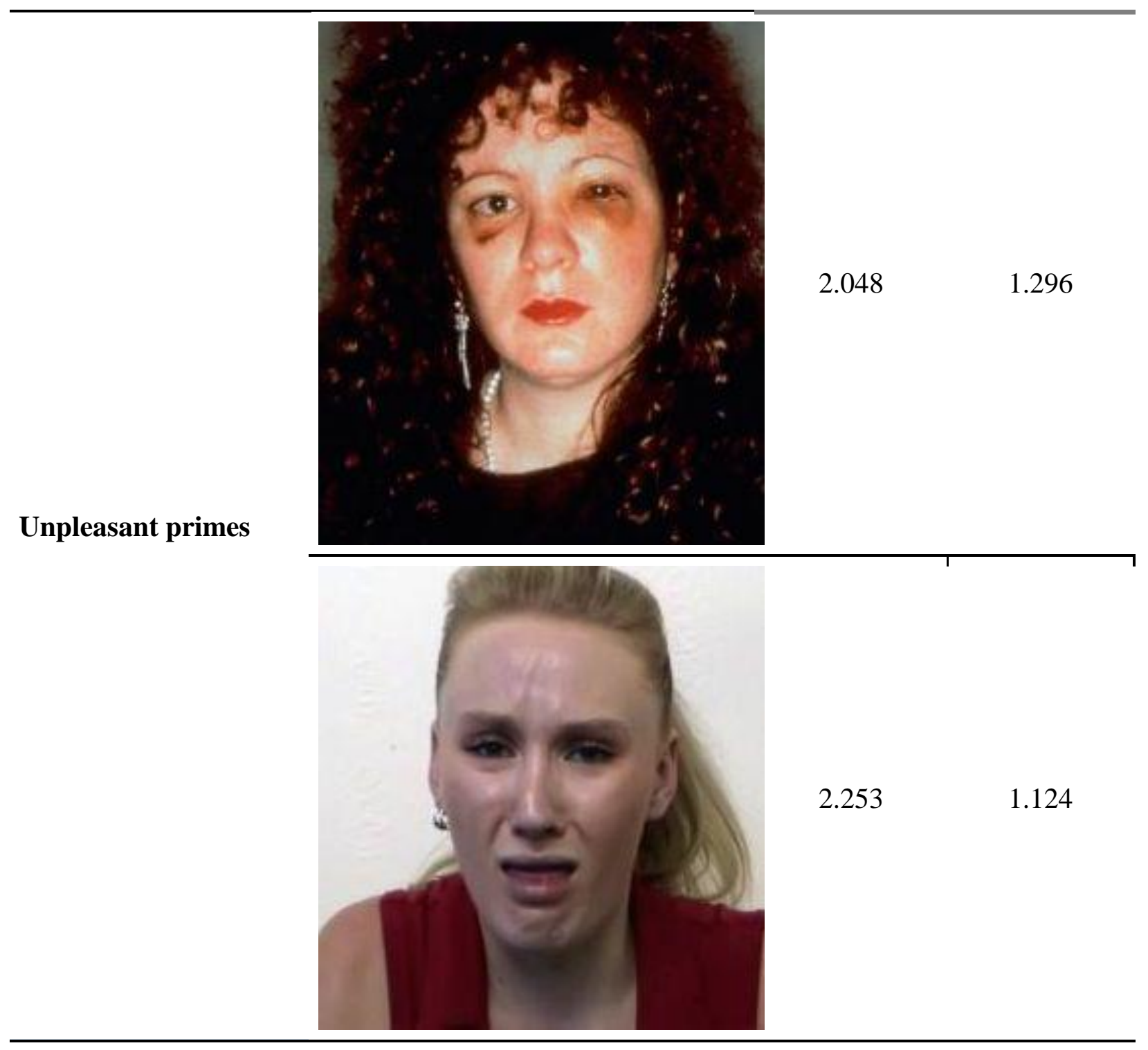




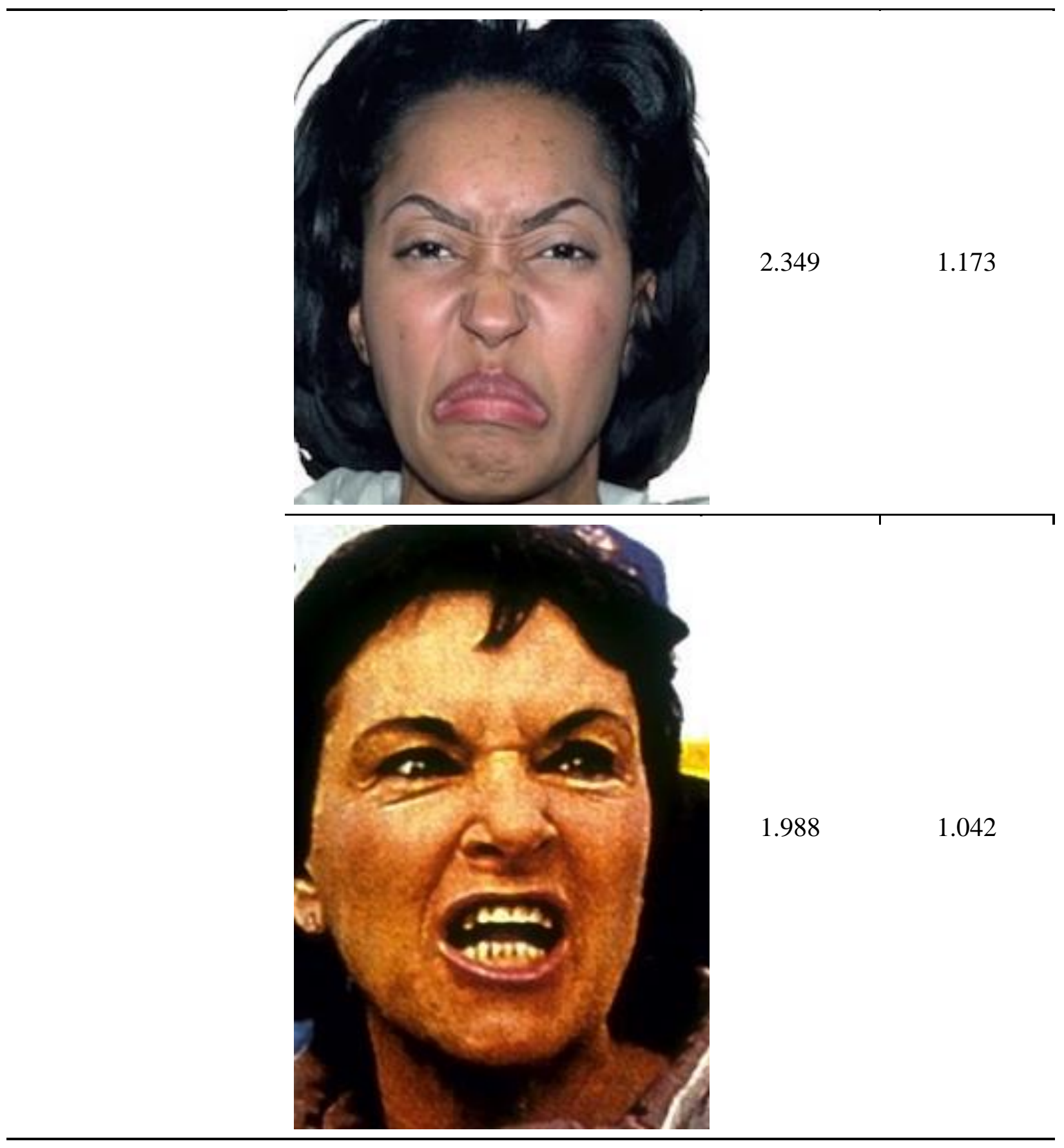




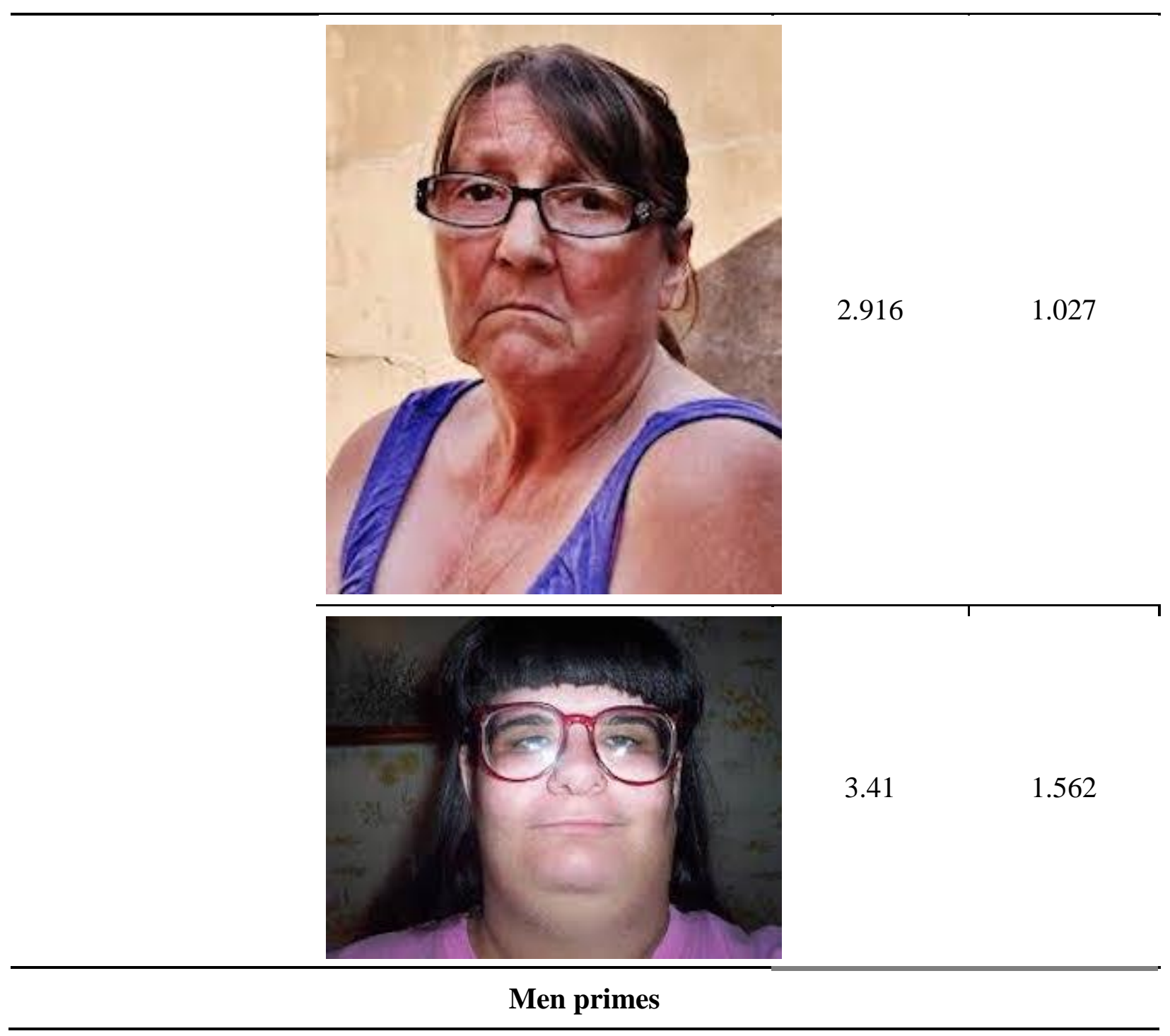




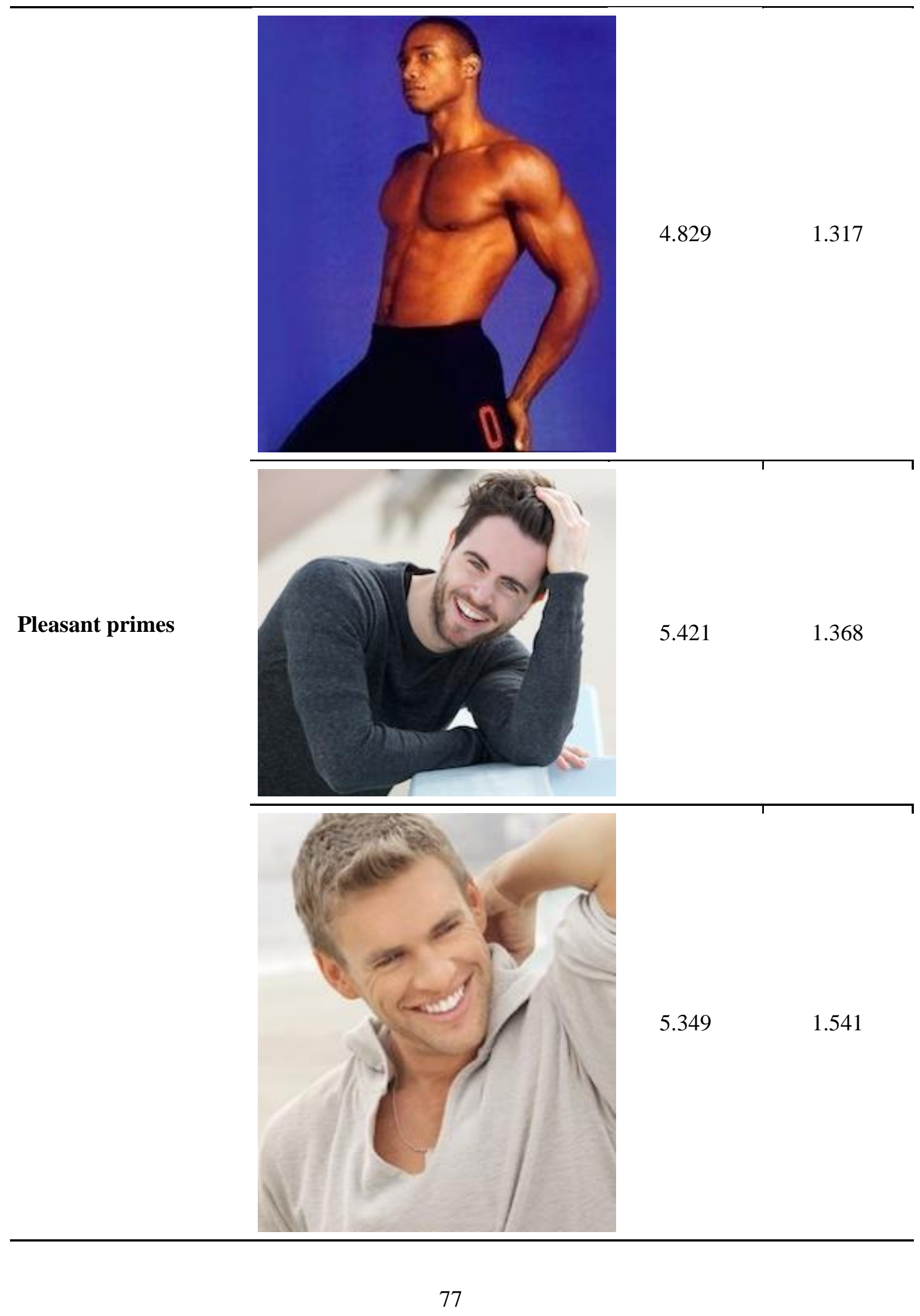




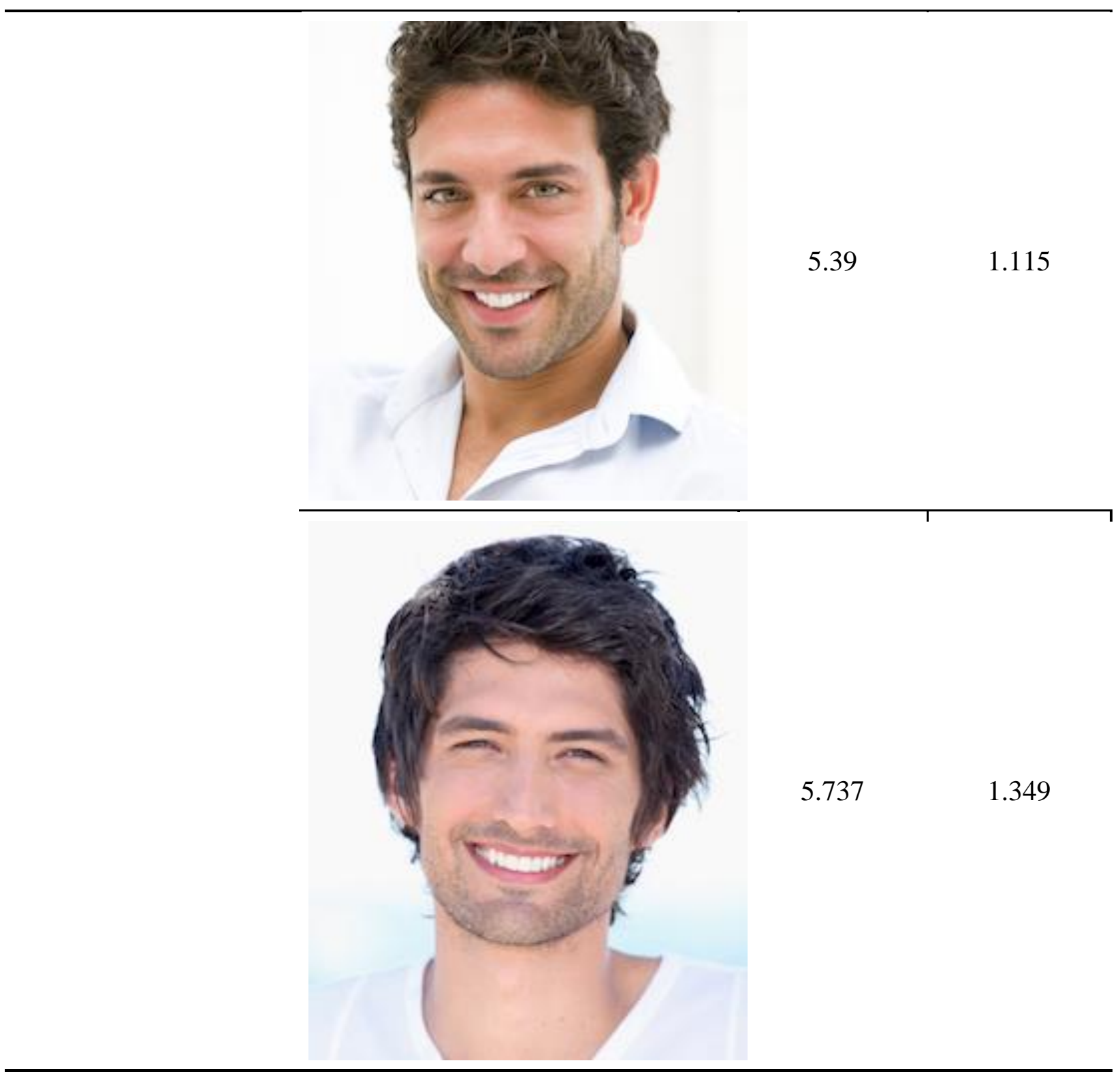




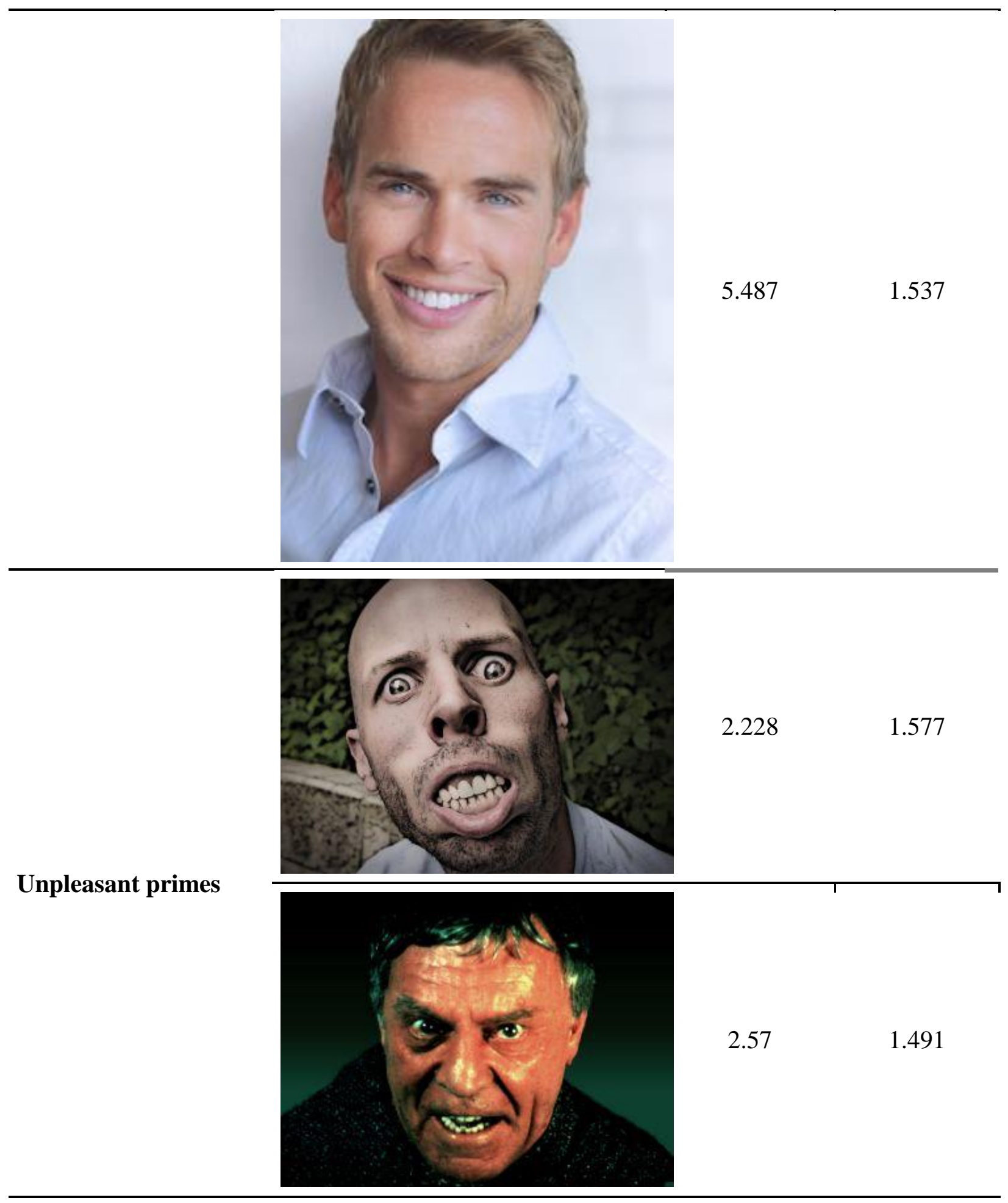




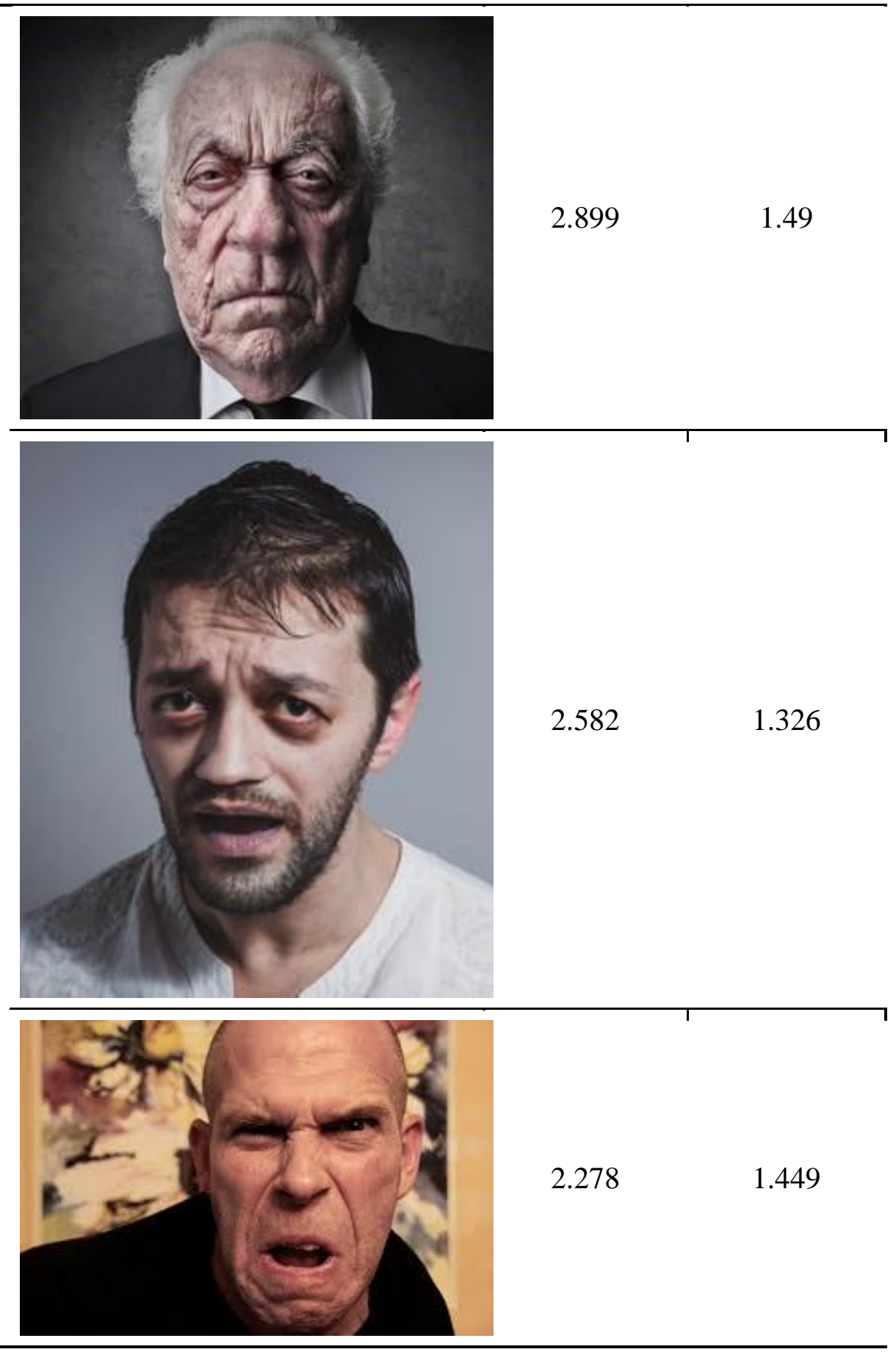




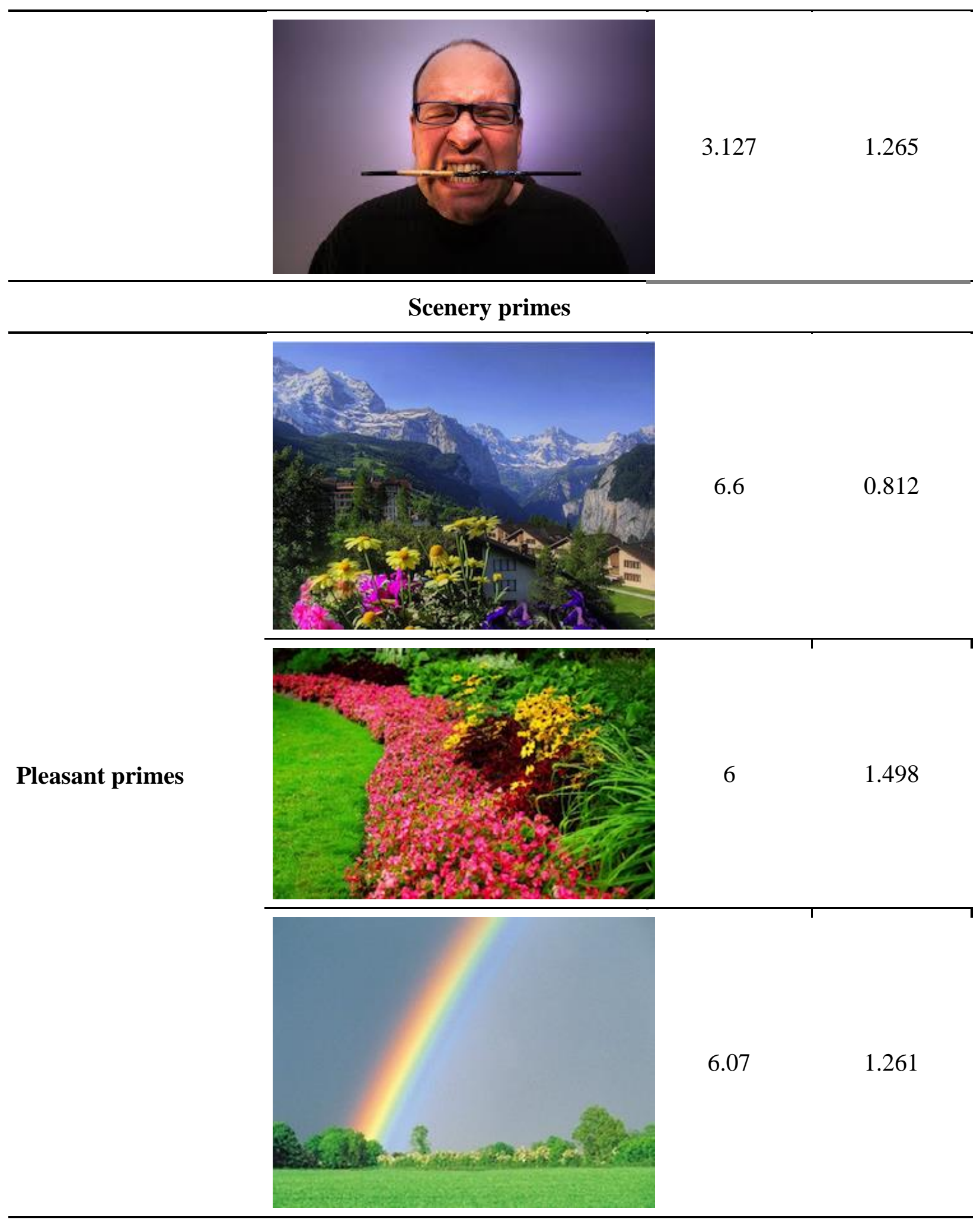




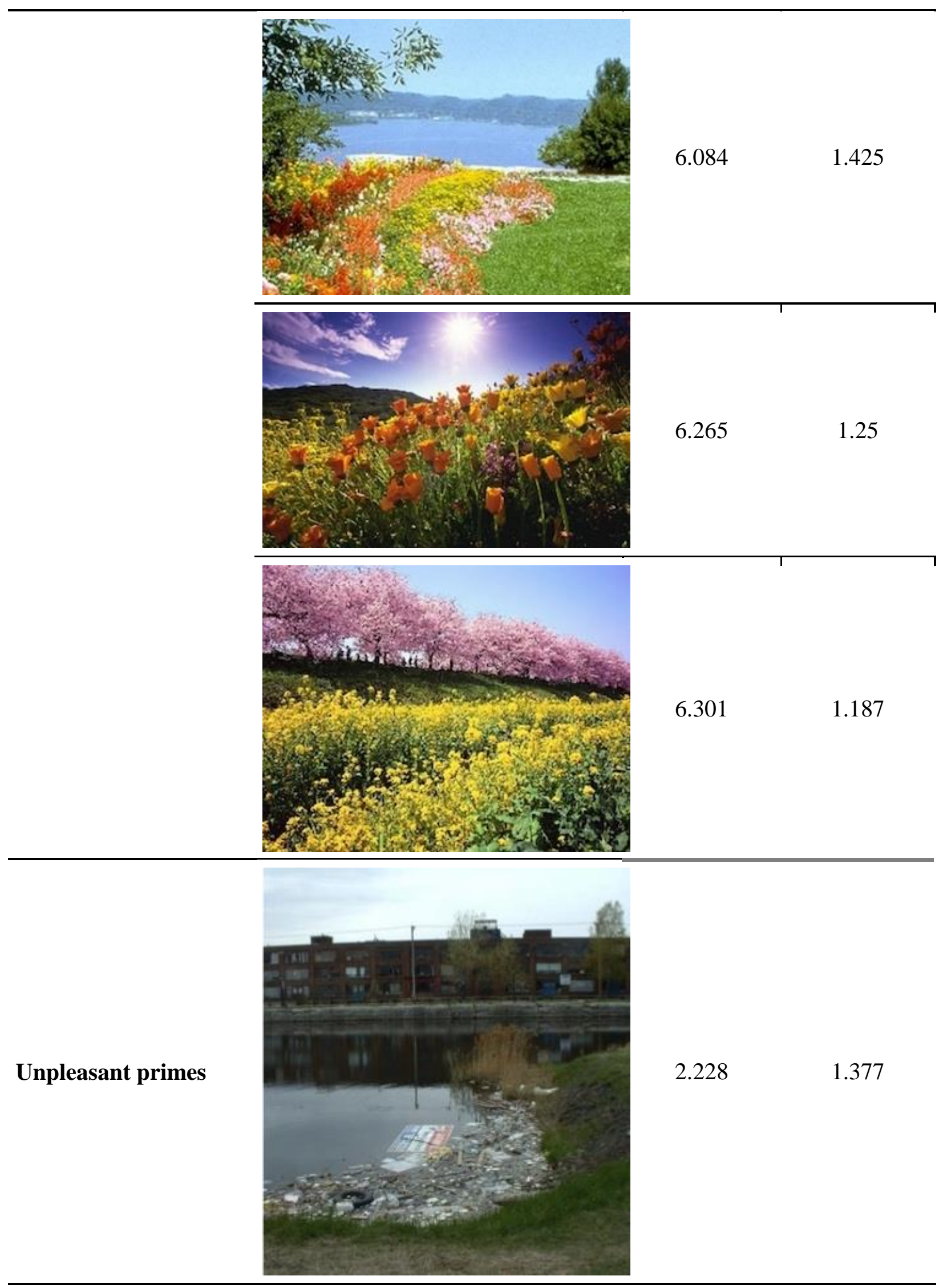



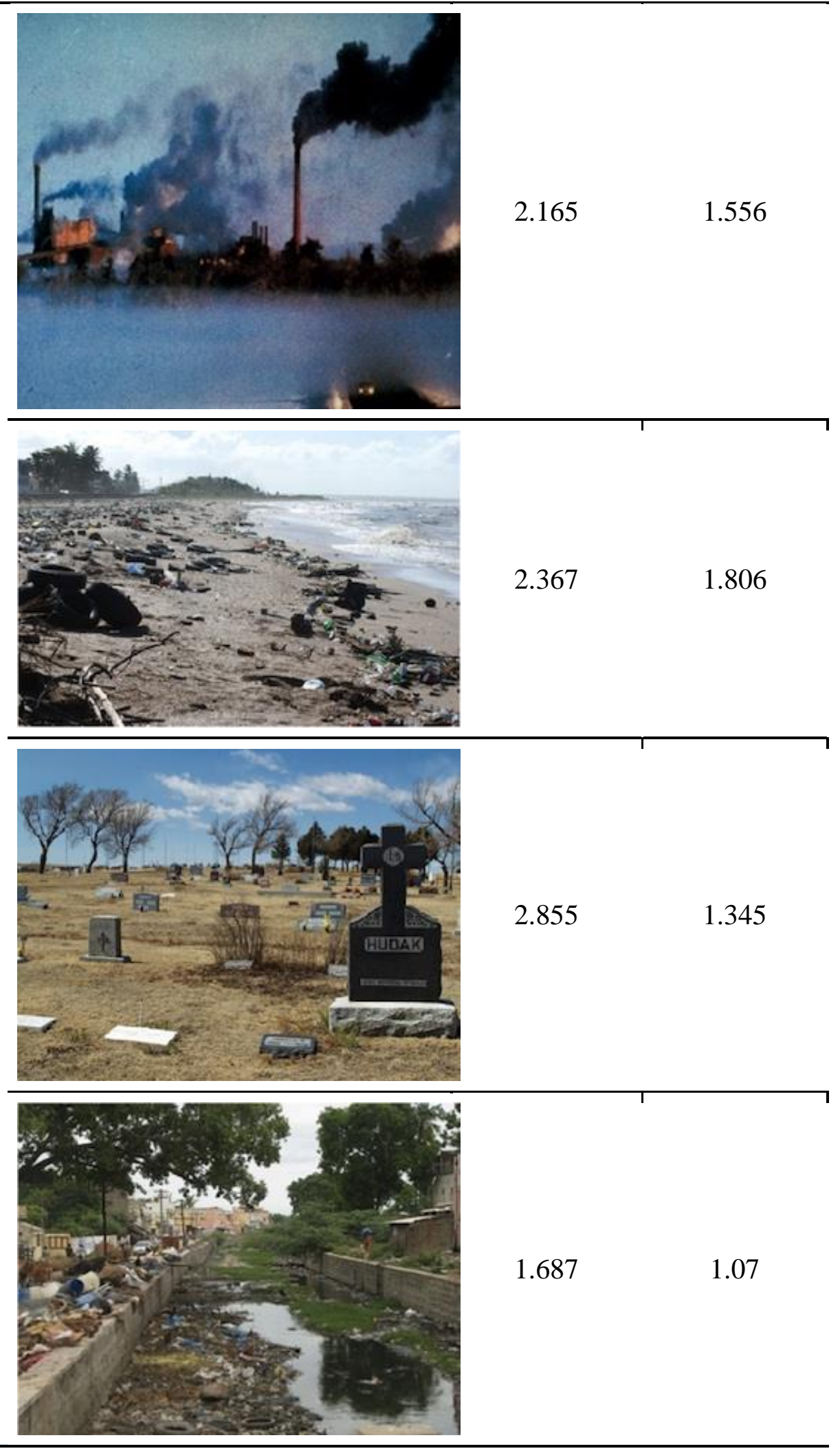


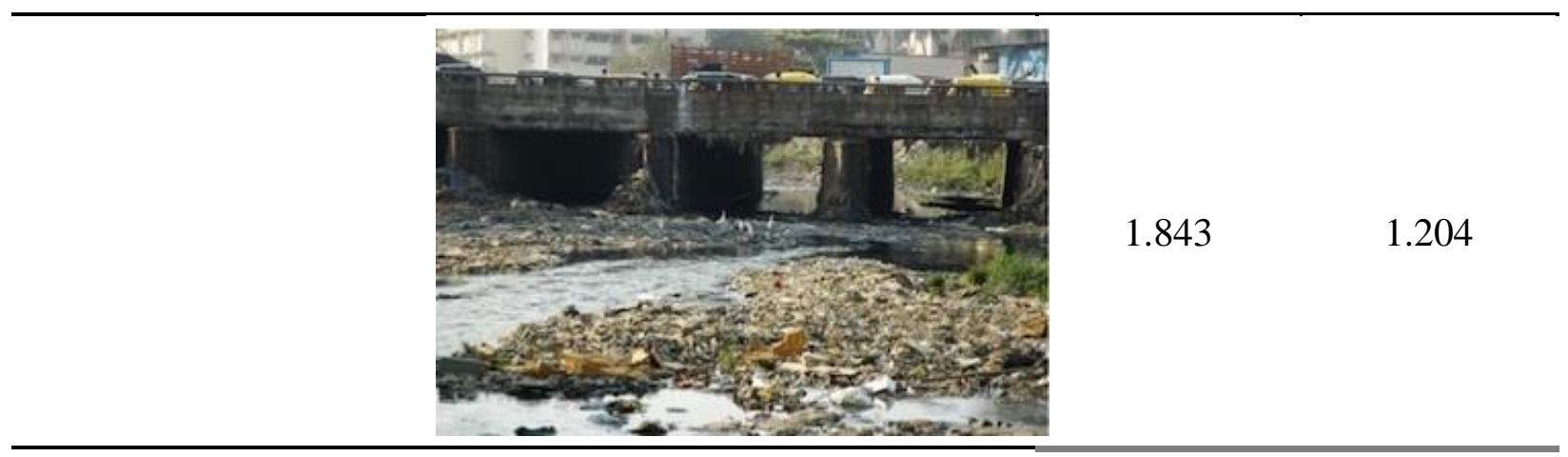

